\title{
Convergence of invariant densities in the small-noise limit
}

\author{
Kevin K. Lin \\ klin@cims.nyu.edu \\ Courant Institute of Mathematical Sciences, New York University \\ 251 Mercer Street, New York, NY 10012
}

July 13, 2004

\begin{abstract}
Let $\rho_{0}$ be an invariant probability density of a deterministic dynamical system $f$ and $\rho_{\epsilon}$ the invariant probability density of a random perturbation of $f$ by additive noise of amplitude $\epsilon$. Suppose $\rho_{0}$ is stochastically stable in the sense that $\rho_{\epsilon} \rightarrow \rho_{0}$ as $\epsilon \rightarrow 0$. Through a systematic numerical study of concrete examples, I show that:

1. The rate of convergence of $\rho_{\epsilon}$ to $\rho_{0}$ as $\epsilon \rightarrow 0$ is frequently governed by power laws: $\left\|\rho_{\epsilon}-\rho_{0}\right\|_{1} \sim \epsilon^{\gamma}$ for some $\gamma>0$.

2. When the deterministic system $f$ exhibits exponential decay of correlations, a simple heuristic can correctly predict the exponent $\gamma$ based on the structure of $\rho_{0}$.

3. The heuristic fails for systems with some "intermittency," i.e. systems which do not exhibit exponential decay of correlations. For these examples, the convergence of $\rho_{\epsilon}$ to $\rho_{0}$ as $\epsilon \rightarrow 0$ continues to be governed by power laws but the heuristic provides only an upper bound on the power law exponent $\gamma$.

Furthermore, this numerical study requires the computation of $\left\|\rho_{\epsilon}-\rho_{0}\right\|_{1}$ for $1.5-2.5$ decades of $\epsilon$ and provides an opportunity to discuss and compare standard numerical methods for computing invariant probability densities in some depth.
\end{abstract}

\section{Introduction}

Deterministic chaotic systems generally possess large numbers of invariant probability measures. Physical considerations lead naturally to the study of invariant measures which are stable under small random perturbations. (See §1.1.) This paper explores the degree of stability of such stochastically stable invariant measures in the setting of discrete-time systems with invariant probability densities.

More precisely, consider dynamical systems of the form

$$
x_{k+1}=f\left(x_{k}\right)
$$

defined by a map $f: M \circlearrowleft$, for example the map $x \mapsto 2 x+a \sin (2 \pi x)(\bmod 1)$ shown in Figure 1 . In this paper, the space $M$ wil always be the circle $S^{1}$, an interval in $\mathbb{R}$, or a product of such sets. Thus operations like addition make 
sense in $M$ and there is always a natural reference measure on $M$, namely the normalized Lebesgue measure. Random perturbations of (1) can therefore be defined via Markov chains of the form

$$
x_{k+1}=f\left(x_{k}\right)+\epsilon \xi_{k}
$$

where the $\left(\xi_{k}\right)$ are independent, identically-distributed random variables with a common probability density independent of $x_{k}$. If $f$ is expanding (i.e. if the singular values of $D f(x)$ are all $>1$ ) for a sufficiently large set of $x \in M$ and $M$ is connected, then the map $f$ possesses a unique invariant probability density $\rho_{0}$ (see $[1,2]$ for precise statements of known results). An invariant density $\rho_{0}$ is stochastically stable if, for every continuous observable $\phi$,

$$
\lim _{\epsilon \rightarrow 0} \int_{M} \phi \rho_{\epsilon}=\int_{M} \phi \rho_{0}
$$

where $\int_{M}$ denotes integration with respect to Lebesgue measure on $M$. When $\rho_{0}$ is stochastically stable, it is natural to try to predict the rate with which $\rho_{\epsilon}$ converges to $\rho_{0}$ as $\epsilon \rightarrow 0$. As the examples in this paper demonstrate, the rate of convergence of $\rho_{\epsilon} \rightarrow \rho_{0}$ can depend on the detailed properties of $\rho_{0}$ and $f$ and may not always be immediately apparent.

As a first step to understanding the factors which can affect the rate of convergence in the limit $\epsilon \rightarrow 0$, this paper systematically examines five concrete examples numerically. It is found that:

1. The rate of convergence of $\rho_{\epsilon}$ to $\rho_{0}$ as $\epsilon \rightarrow 0$ is often governed by power laws: $\left\|\rho_{\epsilon}-\rho_{0}\right\|_{1} \sim \epsilon^{\gamma}$ for some $\gamma>0$, where $\left\|\rho_{\epsilon}-\rho_{0}\right\|_{1}=\int_{M}\left|\rho_{\epsilon}-\rho_{0}\right|$ denotes the $L^{1}$ norm on $M$. Note that because

$$
\left\|\rho_{\epsilon}-\rho_{0}\right\|_{1}=\sup _{\left\{\phi:\|\phi\|_{\infty} \leq 1\right\}} \int_{M} \phi \cdot\left(\rho_{\epsilon}-\rho_{0}\right),
$$

convergence in $L^{1}$ norm implies a uniform rate of convergence of expectation values and is more stringent than stochastic stability.

2. A simple heuristic allows one to predict the exponent $\gamma$ based on the structure of the density $\rho_{0}$, in a sense to be made more precise below (see Equations (7) and (8)).

3. The heuristic fails for systems with some "intermittency," i.e. systems which do not exhibit exponential decay of correlations $[15,17]$. For these examples, the convergence of $\rho_{\epsilon}$ to $\rho_{0}$ continues to be governed by power laws but the heuristic provides only an upper bound on the power law exponent $\gamma$.

The notation " $f \sim g$ " means that there exist positive constants $c_{1}$ and $c_{2}$ such that $c_{1} f(x) \leq g(x) \leq c_{2} f(x)$. Similarly, the notation " $f \lesssim g$ " will be used below to mean that there exists $c>0$ such that $f(x) \leq c g(x)$.

Explaining the heuristic estimate requires the formalism of Perron-Frobenius transfer operators [1]. Let $q$ be a function on $M$ and define the operator $T_{f}$ by

$$
\left(T_{f} q\right)(x)=\sum_{y \in f^{-1}(x)} \frac{q(y)}{|\operatorname{det}(D f(y))|} .
$$

The operator $T_{f}$ reformulates the dynamics in terms of probability densities: if $q$ is the probability density of $x_{k}$, then $T_{f} q$ is the probability density of $x_{k+1}=f\left(x_{k}\right)$. This elementary fact follows from the change of variables formula. Clearly, $\rho_{0}$ is an invariant density of $f$ if and only if it is an eigenfunction of $T_{f}$ with eigenvalue $1: T_{f} \rho_{0}=\rho_{0}$. 
Furthermore, $T_{f}^{n} q \rightarrow \rho_{0}$ as $n \rightarrow \infty$ if the eigenvalue 1 of $T_{f}$ is simple and the initial density $q$ is sufficiently regular. And, if $T_{f}$ has a spectral gap, that is if the eigenvalue 1 is an isolated point of the spectrum $\sigma\left(T_{f}\right)$ of $T_{f}$ and the radius $\left|\sigma\left(T_{f}\right) \backslash\{1\}\right|$ of the smallest disc in $\mathbb{C}$ containing $\sigma\left(T_{f}\right) \backslash\{1\}$ is strictly less than 1 , then the convergence of $T_{f}^{n} q$ to $\rho_{0}$ as $n \rightarrow \infty$ is exponentially fast. The spectral gap condition also implies the exponential decay of correlations, i.e. there exist positive constants $c$ and $\theta<1$ such that

$$
\left|\int_{M} \phi \cdot\left(T_{f}^{n} \psi\right) \cdot \rho_{0}-\int_{M} \phi \rho_{0} \cdot \int_{M} \psi \rho_{0}\right| \leq c \theta^{n}
$$

for all sufficiently smooth observables $\phi$ and $\psi$. Systems with exponential decay of correlations are also said to be exponentially mixing. Note that $1>\theta>\left|\sigma\left(T_{f}\right) \backslash\{1\}\right|$. See [1,2] for details.

The "noisy" transfer operator is defined in a similar way: let $G_{\epsilon}$ be the averaging operator

$$
\left(G_{\epsilon} q\right)(x)=\epsilon^{-d} \int_{M} g\left(\frac{x-y}{\epsilon}\right) q(y) d y,
$$

where $g$ is the common probability density of the IID random variables $\xi_{k}$ and $d=\operatorname{dim}(M)$. The operator $G_{\epsilon}$ represents the effect of additive noise on a probability density $q$. The noisy Perron-Frobenius operator $T_{\epsilon}$ is then

$$
T_{\epsilon}=G_{\epsilon} T_{f}
$$

As before, the operator $T_{\epsilon}$ describes the random dynamics (2) in terms of probability densities. A density $\rho_{\epsilon}$ is invariant under (2) if and only if it is an eigenfunction of $T_{\epsilon}$ of eigenvalue 1.

We can now discuss the heuristic. Under fairly general conditions, $T_{\epsilon}^{n} q$ converges to $\rho_{\epsilon}$ exponentially fast as $n \rightarrow \infty$ for fixed $\epsilon$ [2]. Suppose $\rho_{\epsilon} \rightarrow \rho_{0}$ as $\epsilon \rightarrow 0$. Since $\lim _{n \rightarrow \infty} T_{\epsilon}^{n} \rho_{0}=\rho_{\epsilon}$ exponentially fast, we may expect $\left\|T_{\epsilon}^{n} \rho_{0}-\rho_{\epsilon}\right\|_{1}$ to be small for $n$ finite but sufficiently large. If such an $n$ can be chosen independent of $\epsilon$, it is then natural to guess that for all sufficiently small $\epsilon$,

$$
\left\|\rho_{\epsilon}-\rho_{0}\right\|_{1} \sim\left\|T_{\epsilon}^{n} \rho_{0}-\rho_{0}\right\|_{1}
$$

Equation (7) states that one can estimate the difference between $\rho_{\epsilon}$ and $\rho_{0}$ by studying the effect of applying $T_{\epsilon}$ a finite number of times to the noiseless invariant density $\rho_{0}$. Note that the rate of convergence of $T_{\epsilon}^{n} \rho_{0}$ to $\rho_{\epsilon}$ as $n \rightarrow \infty$ is governed by the size of the spectral gap $\left|\sigma\left(T_{\epsilon}\right) \backslash\{1\}\right|$. If $\left|\sigma\left(T_{\epsilon}\right) \backslash\{1\}\right|$ can become arbitrarily small as $\epsilon \rightarrow 0, n$ may have to be very large (or even increase as $\epsilon$ decreases) in order for (7) to hold. On the other hand, in exponentially mixing systems with a sufficiently large spectral gap $\left|\sigma\left(T_{\epsilon}\right) \backslash\{1\}\right|(\epsilon \geq 0)$, it is likely that one can take $n$ to be a small integer independent of $\epsilon$. Equation (7) should be taken only as a rough guideline for what can be expected in studying the convergence of $\rho_{\epsilon}$ to $\rho_{0}$ in the $L^{1}$ norm in the small noise limit $\epsilon \rightarrow 0$.

In the exponentially mixing examples of Section 2 , it is found empirically that $n=1$ suffices:

$$
\left\|\rho_{\epsilon}-\rho_{0}\right\|_{1} \sim\left\|T_{\epsilon} \rho_{0}-\rho_{0}\right\|_{1}=\left\|G_{\epsilon} \rho_{0}-\rho_{0}\right\|_{1} .
$$

This is not unexpected if $\sup _{0 \leq \epsilon<\epsilon_{0}}\left|\sigma\left(T_{\epsilon}\right) \backslash\{1\}\right| \ll 1$ for some $\epsilon_{0}>0$. Equation (8) states that one can estimate the difference between $\rho_{\epsilon}$ and $\rho_{0}$ by studying the effect of applying the averaging operator $G_{\epsilon}$ once to the noiseless invariant density $\rho_{0}$. Note that "half" of (8) is always true: because $\left(I-T_{\epsilon}\right)\left(\rho_{\epsilon}-\rho_{0}\right)=G_{\epsilon} \rho_{0}-\rho_{0}$, the inequality

$$
\left\|G_{\epsilon} \rho_{0}-\rho_{0}\right\|_{1} \leq 2\left\|\rho_{\epsilon}-\rho_{0}\right\|_{1}
$$




\begin{tabular}{|c|c|c|}
\hline \multirow{2}{*}{ Map } & \multicolumn{2}{|c|}{ Exponent $\gamma=\lim _{\epsilon \rightarrow 0} \frac{\log \left(\left\|\rho_{\epsilon}-\rho_{0}\right\|_{1}\right)}{\log (\epsilon)}$} \\
\cline { 2 - 3 } & Predicted & Computed \\
\hline \hline Uniformly expanding & 2 & $1.996 \pm 0.0071$ \\
\hline Piecewise expanding & 1 & $0.97 \pm 0.033$ \\
\hline Quadratic (Misiurewicz) & $\frac{1}{2}$ & $0.52 \pm 0.056$ \\
& & $0.46 \pm 0.050$ \\
\hline & $\leq 0.5$ & $0.31 \pm 0.028$ \\
Neutral fixed point & $\leq 0.7$ & $0.53 \pm 0.056$ \\
& $\leq 0.3$ & $0.17 \pm 0.033$ \\
\hline Stadium & $\leq 2$ & $1.40 \pm 0.022$ \\
\hline
\end{tabular}

Table 1: Summary of results. The first example, a smooth uniformly expanding map, has a smooth invariant density. Thus $G_{\epsilon} \rho_{0}-\rho_{0}=\frac{1}{6} \epsilon^{2} \rho_{0}^{\prime \prime}+o\left(\epsilon^{2}\right)$ and the heuristic predicts $O\left(\epsilon^{2}\right)$ convergence as $\epsilon \rightarrow 0$. The second example has a piecewise continuous invariant density. The heuristic thus predicts $O(\epsilon)$ convergence. The third example has an invariant density which contain singularities of the form $x^{-1 / 2}$. The heuristic thus predicts $\epsilon^{1 / 2}$ convergence. The last two examples are not exponentially mixing, and the heuristic only predicts upper bounds.

always holds. So as $\epsilon \rightarrow 0,\left\|\rho_{\epsilon}-\rho_{0}\right\|_{1}$ cannot converge to 0 faster than $\left\|G_{\epsilon} \rho_{0}-\rho_{0}\right\|_{1}$ and the heuristic always provides an upper bound on the power law exponent $\gamma$.

In problems where (8) applies, the effect of $G_{\epsilon}$ on $\rho_{0}$ (and hence the scaling of $\left\|G_{\epsilon} \rho_{0}-\rho_{0}\right\|_{1}$ as $\epsilon \rightarrow 0$ ) depends on the structure of $\rho_{0}$ : if $\rho_{0}$ is very smooth, as in the uniformly expanding map in $\S 2.1$, then the effect of $G_{\epsilon}$ will be to simply "flatten" $\rho_{0}$, i.e. decrease the size of $\rho_{0}^{\prime}$ (see Figure 2). The convergence of $G_{\epsilon} \rho_{0}$ to $\rho_{0}$ as $\epsilon \rightarrow 0$ will then be fast (e.g. $O\left(\epsilon^{2}\right)$ ), so the heuristic also predicts a fast convergence of $\rho_{\epsilon}$ to $\rho_{0}$ as $\epsilon \rightarrow 0$. On the other hand, if $\rho_{0}$ contains any discontinuities or singularities (as in the examples of $\S 2.2-\S 3.1$ ), then the main effect of $G_{\epsilon}$ will be to smooth out the discontinuities or singularities. The rate of convergence predicted by the heuristic thus depends on the precise form of the singularity in question. As the heuristic is motivated by the exponential convergence of $T_{\epsilon}^{n} \rho_{0}$ to $\rho_{\epsilon}$ (in the limit $n \rightarrow \infty$ ) it is natural expect it to make sense only when $\left|\sigma\left(T_{\epsilon}\right) \backslash\{1\}\right|<1$ uniformly in $\epsilon \geq 0$. This suggests, among other things, that the heuristic will work only when $T_{f}$ itself has a spectral gap, or that correlations decay exponentially fast in the noiseless system (1). As will be seen, the available numerical evidence supports this claim.

This paper examines five concrete examples, three of which are exponentially mixing (see $\S 2$ ) and two which are not (§3). It is found that the heuristic is valid for each of the exponentially mixing examples but does not work for the intermittent examples. Each example is accompanied by an explanation of the numerical techniques used to compute the invariant densities. Table 1 summarizes the numerical results described in the rest of this paper.

The remainder of this section is devoted to a brief review of background and motivation for this work.

\subsection{Motivation \& background}

Chaotic systems are intrinsically unpredictable. The tools of ergodic theory and statistical mechanics are therefore necessary for questions concerning the long-time behavior of dynamical systems [7]. Invariant probability measures 
capture the long-time statistical properties of dynamical systems.

Deterministic dynamical systems generally possess a multitude of invariant probability measures. For example, fixed points and periodic orbits support invariant measures. However, when the system in question is chaotic, most of these invariant measures are unstable and do not have directly observable effects on the long-time dynamics. The notion of stochastic stability (due to Kolmogorov) arises naturally as a criterion for separating these unstable invariant measures from those which are physically relevant: because no real physical system can be completely isolated from the rest of the universe, every physical experiment is susceptible to the effects of noise. Thus only stochastically stable invariant measures have directly observable effects on the long-term statistics of dynamical systems.

One of the earliest general results on the stochastic stability of invariant measures concerns systems with uniformly hyperbolic attractors. Such systems possess Sinai-Ruelle-Bowen (SRB) measures [21], which (among their many properties) capture the statistical properties of a set of initial conditions of positive Lebesgue measure. Kifer proved that SRB measures of uniformly hyperbolic systems are stochastically stable [11]. However, his method of proof cannot address the question of convergence rate in the limit of small noise. In [2], Baladi and Young examine the question of stochastic stability and the rate of convergence in the setting of expanding maps and convolution-type perturbations. Blank and Keller, in subsequent work, proved results for more general perturbations of 1-dimensional maps [3].

In addition to understanding more deeply the stochastic stability of invariant measures, the results described here may be relevant for numerical studies of dynamical systems with intermittent, metastable behavior. The injection of noise into a numerical simulation can help reduce initialization bias in numerical computations of expectation values via time averaging while adding controllable errors to the computed expectation value [14].

\section{Exponentially mixing systems}

The examples in this section have one common feature: their transfer operators $T_{f}$ all have spectral gaps. That is, $\left|\sigma\left(T_{f}\right) \backslash\{1\}\right|<1$. This implies the exponential decay of correlations. The heuristic estimate (7) or its variant (8) are expected to work for maps whose transfer operators have spectral gaps. This is found to be the case. For all the examples in this section, the common density $g: \mathbb{R} \rightarrow \mathbb{R}$ of the random variables $\xi_{k}$ is taken to be

$$
g(x)=\left\{\begin{array}{cc}
\frac{1}{2}, & |x| \leq 1 \\
0, & |x|>1
\end{array}\right.
$$

(The first two examples are maps on $S^{1}$, here identified with the interval $[0,1]$. The random perturbations should therefore be taken modulo 1.) The perturbations $\epsilon \xi_{k}$ are thus uniform random variables on the interval $[-\epsilon,+\epsilon]$. Calculations using gaussian kernels wrapped around the circle (not shown here) indicate that the scaling is insensitive to the exact form of the kernel $g$.

\subsection{Smooth expanding circle map}

Consider $f: S^{1} \circlearrowleft$ defined by

$$
f(x)=2 x+a \sin (2 \pi x)(\bmod 1) .
$$

See Figure 1. This map is clearly smooth and is uniformly expanding for $0<a<\frac{1}{2 \pi}$. Its invariant density $\rho_{0}$ is easy to compute by discretizing the Perron-Frobenius operator $T_{f}$ on a uniform mesh and is shown in Figure 2 . 


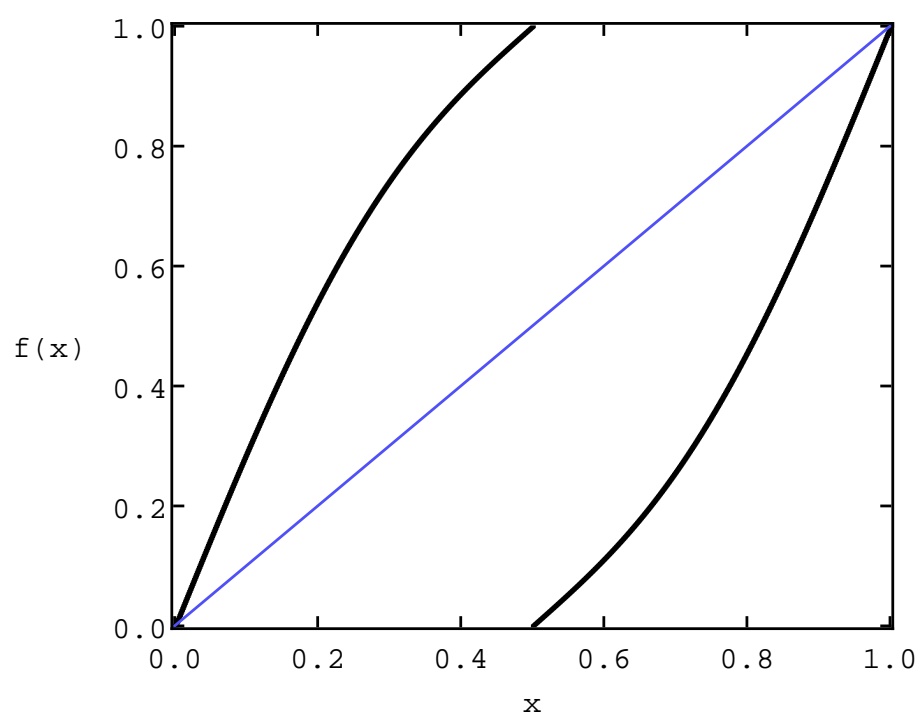

Figure 1: A smooth uniformly expanding map.

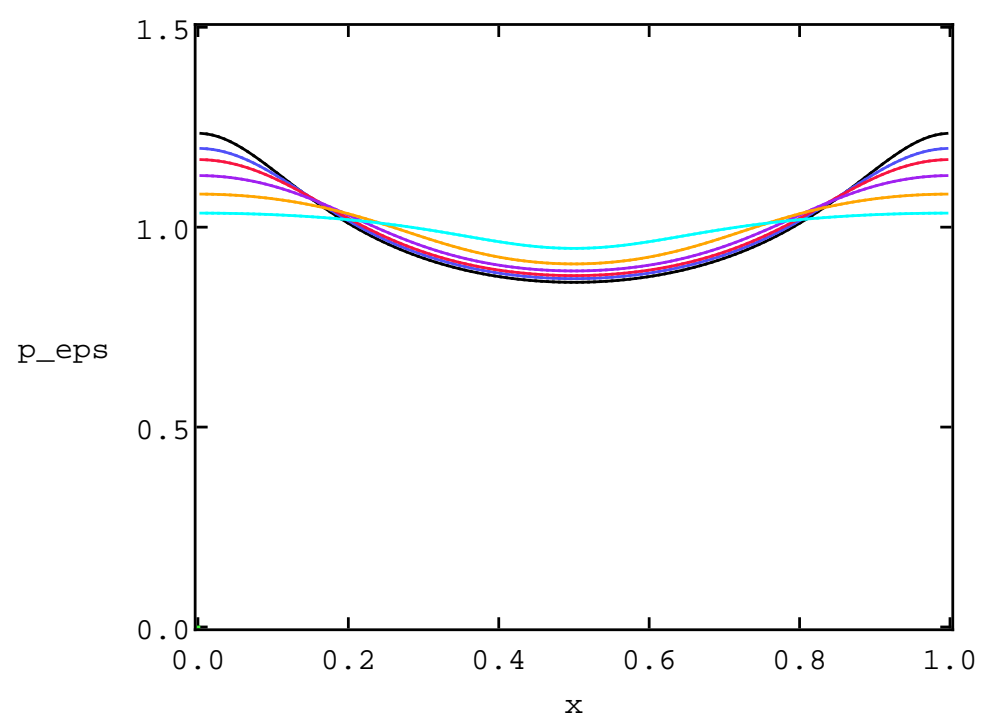

Figure 2: The invariant densities $\rho_{\epsilon}$ with $\epsilon \in\left\{0, \frac{1}{10}, \frac{1}{10 \sqrt{2}}, \frac{1}{5}, \frac{1}{5 \sqrt{2}}, \frac{2}{5}\right\}$, for the map (11). The black curve is $\rho_{0}$; the densities $\rho_{\epsilon}$ become flatter as $\epsilon$ increases. The map parameter is $a=0.15$. 
Before discussing the details of numerical calculations, it should be noted that one can prove (8) for the map (11) when the parameter $a$ is sufficiently small. Although the analysis is simple and uses only standard techniques, it is nevertheless instructive and is included here for completeness. Let $B$ denote the space $C^{1}$ with the usual norm $\|h\|_{B}=\max \left(\|h\|_{\infty},\left\|h^{\prime}\right\|_{\infty}\right)$, and let $B_{0}$ be the subspace $\left\{h \in B: \int_{M} h=0\right\}$. Note that $B_{0}$ is $T_{\epsilon}$-invariant for $\epsilon \geq 0$. It is not difficult to show that the Perron-Frobenius operator $T_{f}$ associated with (11) has the property that $\left\|T_{f}\right\|_{B_{0}}<1$ when the parameter $a$ is sufficiently small: differentiating (4) shows that $\left\|\left(T_{f} q\right)^{\prime}\right\|_{\infty} \leq c_{a}\|q\|_{B}$ for some constant $c_{a}$ dependent on $a$, and $c_{a}<1$ when $a$ is small. Because $\int_{M} T_{f} q=\int_{M} q=0$, the Poincaré inequality shows that $\left\|T_{f} q\right\|_{\infty} \leq\left\|\left(T_{f} q\right)^{\prime}\right\|_{\infty}$. So $\left\|T_{f}\right\|_{B_{0}}=\sup \left\{\left\|T_{f} q\right\|_{B}: q \in B_{0},\|q\|_{B}=1\right\} \leq c<1$.

Now let $g$ be a probability density on $S^{1}$ and let $G_{\epsilon}$ be the associated averaging operator. Denote the (unique) invariant density of $T_{\epsilon}=G_{\epsilon} T_{f}$ by $\rho_{\epsilon}$. Since $\left\|G_{\epsilon}\right\|_{B} \leq 1,\left\|T_{\epsilon}\right\|_{B_{0}} \leq\left\|T_{f}\right\|_{B_{0}}<1$ for all $\epsilon \geq 0$. Thus the restriction $\left(I-T_{\epsilon}\right) \uparrow_{B_{0}}$ of $I-T_{\epsilon}$ to $B_{0}$ has bounded inverse for all $\epsilon \geq 0$. A simple calculation shows that

$$
\rho_{\epsilon}-\rho_{0}=\left[\left(I-T_{\epsilon}\right)\left\lceil_{B_{0}}\right]^{-1}\left(G_{\epsilon} \rho_{0}-\rho_{0}\right) .\right.
$$

Thus

$$
\begin{aligned}
\left\|\rho_{\epsilon}-\rho_{0}\right\|_{B} & \leq\left\|\left(I-T_{\epsilon}\right)^{-1}\right\|_{B_{0}}\left\|G_{\epsilon} \rho_{0}-\rho_{0}\right\|_{B} \\
& \leq\left(1-\left\|T_{f}\right\|_{B_{0}}\right)^{-1}\left\|G_{\epsilon} \rho_{0}-\rho_{0}\right\|_{B} .
\end{aligned}
$$

Also

$$
\begin{aligned}
\left\|G_{\epsilon} \rho_{0}-\rho_{0}\right\|_{B} & =\left\|\left(I-T_{\epsilon}\right) \cdot\left(I-T_{\epsilon}\right)^{-1} \cdot\left(G_{\epsilon} \rho_{0}-\rho_{0}\right)\right\|_{B} \\
& \leq\left\|I-T_{\epsilon}\right\|_{B_{0}} \cdot\left\|\rho_{\epsilon}-\rho_{0}\right\|_{B} .
\end{aligned}
$$

Since $\left\|I-T_{\epsilon}\right\|_{B_{0}} \leq 1+\left\|T_{f}\right\|_{B_{0}}$, this means

$$
\left\|\rho_{\epsilon}-\rho_{0}\right\|_{B} \sim\left\|G_{\epsilon} \rho_{0}-\rho_{0}\right\|_{B}
$$

with $\epsilon$-independent constants in the $\sim$ relation. Note that this already means $\rho_{\epsilon}-\rho_{0}$ converges to 0 at the same rate as $G_{\epsilon} \rho_{0}-\rho_{0}$ in the $C^{1}$ metric as $\epsilon \rightarrow 0$. Combining Equation (9) and the fact that $\left\|\rho_{\epsilon}-\rho_{0}\right\|\left\|_{B} \geq\right\| \rho_{\epsilon}-\rho_{0} \|_{1}$ yields

$$
\left\|G_{\epsilon} \rho_{0}-\rho_{0}\right\|_{1} \lesssim\left\|\rho_{\epsilon}-\rho_{0}\right\|_{1} \lesssim\left\|G_{\epsilon} \rho_{0}-\rho_{0}\right\|_{B}
$$

For the map (11), it can be shown that the unique invariant density $\rho_{0}$ is smooth (see [1] for details). So $G_{\epsilon} \rho_{0}-\rho_{0}=$ $\frac{1}{6} \epsilon^{2} \rho_{0}^{\prime \prime}+o\left(\epsilon^{2}\right)$ and $\left\|G_{\epsilon} \rho_{0}-\rho_{0}\right\|_{1} \sim\left\|G_{\epsilon} \rho_{0}-\rho_{0}\right\|_{B}$ when $\epsilon$ is sufficiently small, so that

$$
\left\|G_{\epsilon} \rho_{0}-\rho_{0}\right\|_{1} \sim\left\|\rho_{\epsilon}-\rho_{0}\right\|_{1} \sim \epsilon^{2} .
$$

Thus the heurstic estimate holds for (11) when $a$ is small. A little more is true: for any smooth expanding map $f, 1$ is an eigenvalue of the Perron-Frobenius operator $T_{f}$ with algebraic multiplicity 1 (see [1]). This means $T_{f} \uparrow_{B_{0}}$ has spectral radius $<1$. As $\left|\sigma\left(T_{f}\right)\right|=\lim _{n \rightarrow \infty}|| T_{f}^{n}||^{1 / n}$, the facts above together with the previous argument show that for any smooth expanding map $f$ there exists an integer $N \geq 1$ such that the heuristic holds for $f^{N}=f \circ f \circ \ldots \circ f$, i.e. if $\rho_{\epsilon}^{(N)}$ is the invariant density for $x_{k+1}=f^{N}\left(x_{k}\right)+\epsilon \xi_{k}$ then $\left\|\rho_{\epsilon}^{(N)}-\rho_{0}\right\|_{1} \sim\left\|G_{\epsilon} \rho_{0}-\rho_{0}\right\|_{1}$.

To test the general validity of the heuristic (8) when $a$ is not necessarily small, it is natural to choose $a$ close to 


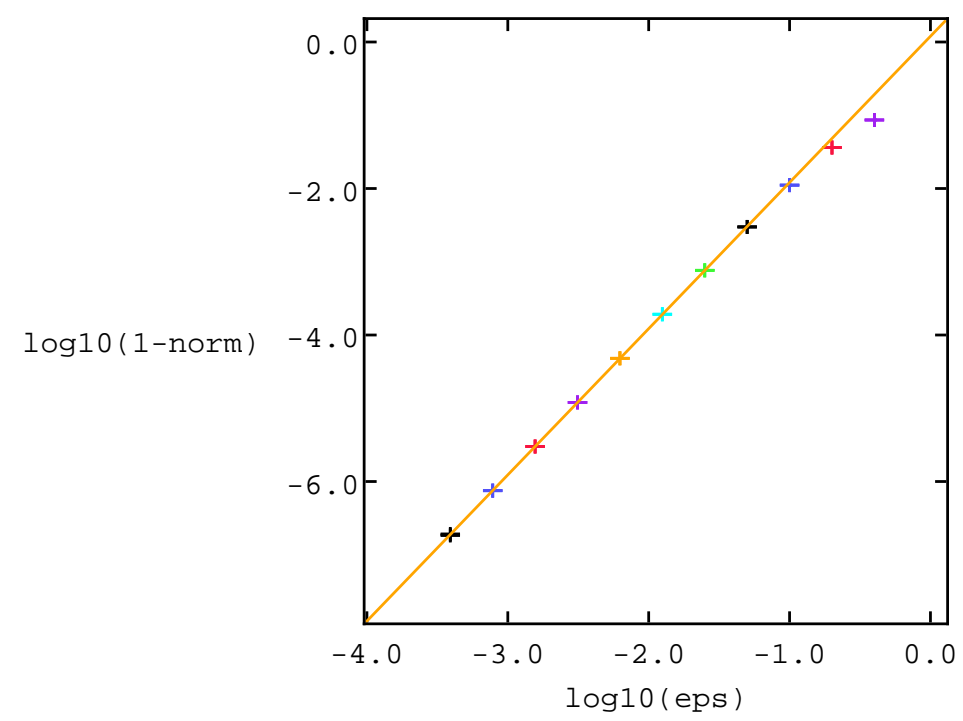

Figure 3: The differences $\left\|\rho_{\epsilon}-\rho_{0}\right\|_{1}$ and $\left\|G_{\epsilon} \rho_{0}-\rho_{0}\right\|_{1}$ as a function of $\epsilon$ on a log-log graph. The slope is $1.996 \pm$ 0.0071 . The slope is calculated using least-squares regression on the interval $\epsilon \in\left[10^{-3.5}, 10^{-1}\right]$. Note that this means data points on the far right are discarded. Throughout the paper, data points for which error bars are available are drawn as vertical lines with 3 horizontal marks: the vertical line marks the abscissa of the data point, the middle mark the ordinate, and the top and bottom marks are the upper and lower bounds on the error. The error bars in this figure are too small to be seen.

$1 / 2 \pi=0.15915494309189535 \ldots$. In what follows, $a$ is set to 0.15 . In Figure 2, it can be seen that $\rho_{\epsilon}$ becomes flatter as $\epsilon$ increases. This is not suprising: as $\epsilon \rightarrow \infty$, the noise dominates the dynamics and the invariant density is just the Lebesgue measure. As $\epsilon \rightarrow 0$, the densities converge to $\rho_{0}$.

The $L^{1}$ distances $\left\|\rho_{\epsilon}-\rho_{0}\right\|_{1}$ are plotted in Figure 3 as a function of $\epsilon$ on a log-log scale. Clearly, $\left\|\rho_{\epsilon}-\rho_{0}\right\|_{1} \sim \epsilon^{\gamma}$ for some $\gamma$. The exponent $\gamma$ can be calculated as the slope of the line in Figure 3 using standard least-squares regression: it is about $1.996 \pm 0.0071$ when $\gamma$ is computed via least-squares regression on the interval $\epsilon \in\left[10^{-3.5}, 10^{-1}\right]$.

In comparison, $G_{\epsilon} \rho_{0}-\rho_{0}=\frac{1}{6} \epsilon^{2} \rho_{0}^{\prime \prime}+o\left(\epsilon^{2}\right)$ implies $\left\|G_{\epsilon} \rho_{0}-\rho_{0}\right\|_{1} \sim \epsilon^{2}$. Thus, the heuristic estimate (8) predicts that

$$
\left\|\rho_{\epsilon}-\rho_{0}\right\|_{1} \sim \epsilon^{2} .
$$

For this particular map, the invariant density $\rho_{0}$ is smooth. Therefore the main effect of $G_{\epsilon}$ on $\rho_{0}$ is to "flatten" it out, consistent with the pictures of $\rho_{\epsilon}$ in Figure 2.

The numerical data thus provide evidence that the heuristic is valid for values of the parameter $a$ which is not close to 0 . The heuristic argument predicts a little bit more than just the exponent $\gamma$ : it predicts that when the spectral gap of $T_{f}$ is sufficiently large, the quantities $\left\|\rho_{\epsilon}-\rho_{0}\right\|_{1}$ and $\left\|G_{\epsilon} \rho-\rho_{0}\right\|_{1}$ will be comparable. This is also borne out by the data, as can be seen in Figure 4. The numerically computed spectral gap is about 0.848 , which is quite large. This explains why $\left\|\rho_{\epsilon}-\rho_{0}\right\|_{1}$ and $\left\|G_{\epsilon} \rho_{0}-\rho_{0}\right\|_{1}$ are so close in Figure 4. 


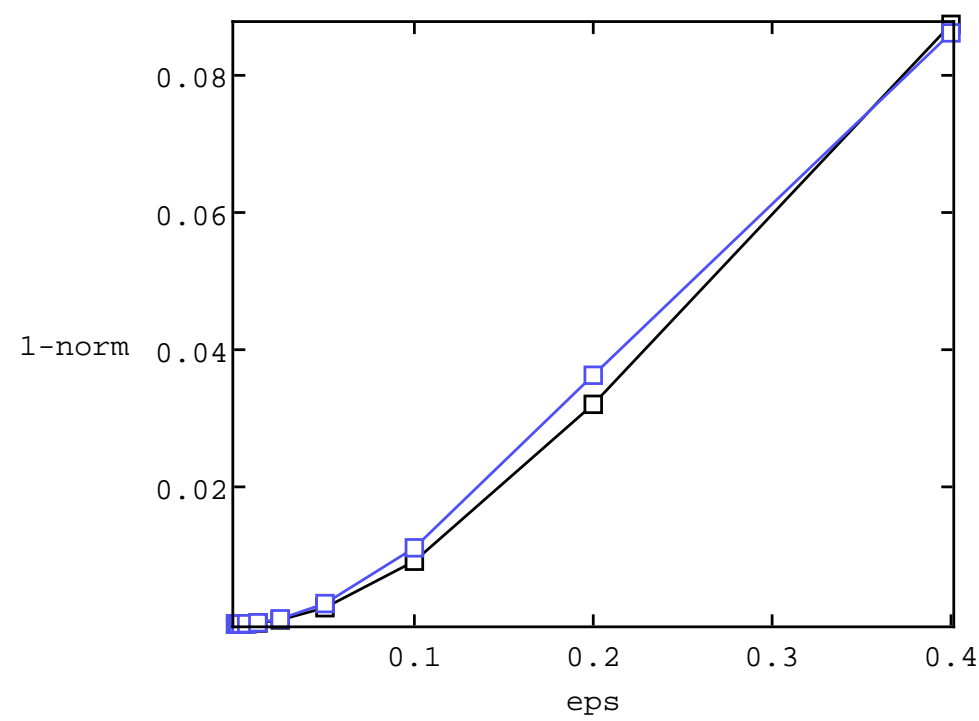

Figure 4: The differences $\left\|\rho_{\epsilon}-\rho_{0}\right\|_{1}$ and $\left\|G_{\epsilon} \rho_{0}-\rho_{0}\right\|_{1}$ as a function of $\epsilon$ on a linear graph.

\section{Numerical method.}

It is straightforward to compute the invariant densities shown in Figure 2 using a finite difference discretization of the Perron-Frobenius operator $T_{f}$. Let $\phi_{j}$ denote the inverse branches of $f: f \circ \phi_{j}=\mathrm{id}, j=1,2$. (There are two branches because the map $f$ is 2-to-1.) We can then write $T_{f}$ as

$$
\left(T_{f} q\right)(x)=\sum_{j=1}^{2} \frac{q\left(\phi_{j}(x)\right)}{\left|f^{\prime}\left(\phi_{j}(x)\right)\right|} .
$$

The numerical procedure is then:

1. Identify $S^{1}$ with the interval $[0,1]$ with periodic boundary conditions and let $\hat{x}_{i}=i / N, i=0,1,2, \ldots, N-1$, be a uniform grid of size $N$.

2. Let $\hat{\rho}$ be an $N$-vector with nonnegative entries. Define the matrix $\hat{T}$ by

$$
(\hat{T} \hat{\rho})_{i}=\sum_{j=1}^{2} \frac{\hat{\rho}\left(\phi_{j}\left(\hat{x}_{i}\right)\right)}{\left|f^{\prime}\left(\phi_{j}\left(\hat{x}_{i}\right)\right)\right|},
$$

where we extend the $N$-vector $\hat{\rho}$ to a function on $[0,1]$ via polynomial interpolation using the grid points closest to $x$. Note that interpolation is necessary becuase the vector $\hat{\rho}$ only contains values of the density on the grid and $\phi_{j}\left(\hat{x}_{i}\right)$ will generally not be a grid point.

3. Using a numerical linear algebra package like ARPACK [13], which implements an iterative Arnoldi eigenproblem solver, compute the eigenvector $\hat{\rho}_{0}$ of $\hat{T}$ with the largest eigenvalue. This requires only a function for performing matrix-vector multiplies, which avoids storing and diagonalizing the entire matrix $\hat{T}$. 
In the computations shown in this section, the polynomial interpolation of $\hat{\rho}$ utilizes a stencil with 6 points and the grid consists of $N=10^{4}$ points. For smooth maps like (11), the order of convergence (as $N \rightarrow \infty$ ) is formally 6 . This is confirmed empirically by numerical tests. Thus the finite difference scheme converges rather rapidly and provides an efficient way to compute $\rho_{0}$.

Note that there is a closely related method, due to Ulam [10], which consists of forming a partition of the phase space and computing transition probabilities to form a stochastic matrix. The stochastic matrix corresponds to a finitestate Markov chain which mimics the dynamics of the map $f$ and whose invariant probability distribution can be computed using numerical eigenproblem solvers. Ulam's method is more general: it applies to dynamical systems whose invariant measures are singular. As a method for computing invariant densities, however, it is empirically found not to be as accurate or efficient as the high-order finite difference schemes outlined above. This is perhaps not so surprising: Ulam's method is, intuitively, only a low-order finite element scheme for the equation $T_{f} \rho_{0}=\rho_{0}$.

The computation of $\rho_{\epsilon}$ relies on a similar finite difference scheme. Instead of discretizing $T_{\epsilon}=G_{\epsilon} T_{f}$ directly, it is easier to reuse the matrix $\hat{T}$ from the computation of $\rho_{0}$ and multiply it from the left by a discretization $\hat{G}$ of $G_{\epsilon}$. The matrix $\hat{G}$ is constructed by

$$
(\hat{G} \hat{\rho})_{i}=\frac{1}{2 \epsilon} \int_{\hat{x}_{i}-\epsilon}^{\hat{x}_{i}+\epsilon} \hat{\rho}(x) d x .
$$

The integral is evaluated numerically using the trapezoid rule. This simple scheme suffices here because it is secondorder accurate, and with $N=2 \times 10^{4}$ the expected error is on the order of $10^{-8}$. This is smaller than the $L^{1}$ norms $\left\|\rho_{\epsilon}-\rho_{0}\right\|_{1}$ to be computed. ARPACK can then be applied to the matrix $\hat{G} \cdot \hat{T}$. The reason that $T_{f}$ is discretized to 6 th order while $G_{\epsilon}$ is only discretized to 2 nd order is empirical: numerical experiments indicate that discretizing $T_{f}$ to higher order accelerates the convergence of ARPACK eigenproblem routines. This phenomenon is not completely understood, but intuitively a higher-order discretization $\hat{T}$ of $T_{f}$ reproduces the spectral properties of $T_{f}$ more accurately. As $T_{f} \uparrow_{B_{0}}$ is strongly contractive, $\hat{T}$ will also be strongly contractive.

In actual computations performed on Sun Ultra 5 workstations and on PowerPC G3/G4-based Macintosh computers, the computation of each invariant density $\rho_{\epsilon}$ can take anywhere from 10 seconds to 5 minutes. The variations in running time are sometimes quite unpredictable; they are certainly not monotic in $\epsilon$ as one might expect. Note that the running time of ARPACK routines depends quite sensitively on the spectral decomposition of $\hat{T}$, so the variations in running times provide some indirect evidence in the complexity of the $\epsilon$-dependence of the spectrum of $T_{\epsilon}$. In any case, the finite difference scheme is in general quite efficient and allows the systematic exploration of different values of $\epsilon$ (shown above) and $a$ (not shown here).

The error bars in Figure 3 are computed by repeating the calculations with $N=10^{4}$ points and checking the numerical convergence of the resulting estimates for $\left\|\rho_{\epsilon}-\rho_{0}\right\|_{1}$. This provides error estimates $\Delta A(\epsilon)$ for each computed value of $A(\epsilon)=\log _{10}\left(\left\|\rho_{\epsilon}-\rho_{0}\right\|_{1}\right)$. These error estimates can then be combined to provide an upper bound on the error $\Delta \gamma$ in the estimated exponent $\gamma$ by linearizing $\gamma$ about the computed values $A\left(\epsilon_{i}\right)$ :

$$
\Delta \gamma \lesssim \frac{1}{n} \sum_{i=1}^{n}\left|\frac{\log _{10}\left(\epsilon_{i}\right)-\mu}{\sigma^{2}} \cdot \Delta A\left(\epsilon_{i}\right)\right|
$$

where $\mu=\frac{1}{n} \sum_{i=1}^{n} \log _{10}\left(\epsilon_{i}\right)$ and $\sigma^{2}=\frac{1}{n} \sum_{i=1}^{n}\left(\log _{10}\left(\epsilon_{i}\right)-\mu\right)^{2}$. Note that because of the high order of convergence of the finite difference scheme, the main source of error in estimating $\left\|r h o_{\epsilon}-\rho_{0}\right\|_{1}$ actually comes from the evaluation of $\left\|\hat{\rho}_{\epsilon}-\hat{\rho}_{0}\right\|_{1}$ using the trapezoid rule. Since $\rho_{\epsilon}-\rho_{0}$ is continuous, the trapezoid rule is second-order, and this error dominates all others in the computation. It is therefore natural to combine the results of the two runs to obtain a more accurate answer via Richardson extrapolation. This has been done in computing the exponent $\gamma$. The error estimates 
are therefore rather conservative.

\subsection{Piecewise-expanding map}

The second example is

$$
f(x)=\left\{\begin{array}{ll}
x+\frac{1}{2}, & 0 \leq x \leq \frac{1}{2} \\
2(1-x), & \frac{1}{2}<x \leq 1
\end{array} .\right.
$$

Strictly speaking, $f$ is not expanding because $f^{\prime}(x)=1$ for $0 \leq x \leq \frac{1}{2}$. However, $f^{2}=f \circ f$ is expanding and $f$ falls within the class of piecewise expanding maps considered by Lasota and Yorke [12].

The invariant density of (19) is easy to compute analytically: it is

$$
\rho_{0}(x)=\left\{\begin{array}{ll}
\frac{2}{3}, & 0 \leq x \leq \frac{1}{2} \\
\frac{4}{3}, & \frac{1}{2}<x \leq 1
\end{array} .\right.
$$

The invariant densities $\rho_{\epsilon}$ are plotted in Figure 5. Again, it can be seen that $\rho_{\epsilon}$ becomes flatter as $\epsilon$ increases: the main effect of $G_{\epsilon}$ is to smear out the jump discontinuities of $\rho_{0}$. Note that the "overshoot" visible in Figure 5 at $x=0$ and $x=\frac{1}{2}$, reminiscent of the Gibbs phenomenon, is not a numerical artifact. Rather it is the product of the action of the map and the random perturbation. More precisely, it is produced by the following mechanism:

1. Consider $T_{\epsilon}^{n} \rho_{0}$ with $n=1,2,3, \ldots$ For $n=1, T_{\epsilon} \rho_{0}=G_{\epsilon} \rho_{0}$ looks very much like $\rho_{0}$, but the action of $G_{\epsilon}$ replaces the discontinuity with a linear transition region of $O(\epsilon)$ width.

2. On the next iterate, the action of $T_{f}$ then cuts $G_{\epsilon} \rho_{0}$ into two pieces and rearranges them in such a way as to produce the "overshoot" of $O(1)$ height and $O(\epsilon)$ width. The next application of $G_{\epsilon}$ smooths out the transition some more but does not change the asymptotic $\epsilon$-dependence of the overshoot and the transition region.

As $T_{f}^{n} \rho_{0}$ converges to $\rho_{\epsilon}$ with increasing $n$, this process is iterated to produce the overshoot structure. Seen in this light, the overshoot must have $O(1)$ height and $O(\epsilon)$ width, making a $O(\epsilon)$ contribution to $\left\|\rho_{\epsilon}-\rho_{0}\right\|_{1}$.

Clearly, the main effect of $G_{\epsilon}$ is to smear out the discontinuity in $\rho_{0}$. So $\left\|G_{\epsilon} \rho_{0}-\rho_{0}\right\|_{1} \sim \epsilon$ and the heuristic predicts

$$
\left\|\rho_{\epsilon}-\rho_{0}\right\|_{1} \sim \epsilon
$$

This is consistent with the densities $\rho_{\epsilon}$ shown in Figure 5. These $L^{1}$ norms are plotted in Figure 6 as a function of $\epsilon$ on a log-log scale: the slope $\gamma$ is $0.97 \pm 0.033$, consistent with the prediction that $\gamma=1$. The linear scaling of $\left\|\rho_{\epsilon}-\rho_{0}\right\|_{1}$ with $\epsilon$ should also be apparent on a linear scale. This is indeed the case: see Figure 7.

The proof from Section 2.1 can be adapted to show that there exists an $N>0$ such that if $\rho_{\epsilon}^{(N)}$ is the unique invariant density of $x_{k+1}=f^{N}\left(x_{k}\right)+\epsilon \xi_{k}$, then $T_{f^{N}}$ is a bounded operator on the space $B$ of functions of bounded total variation with the norm $\|\cdot\|_{B V}=\|\cdot\|_{1}+$ (total variation) and $\left\|T_{f^{N}}\right\|_{B_{0}}<1$. One obtains $\left\|\rho_{\epsilon}-\rho_{0}\right\|_{B} \sim$ $\left\|G_{\epsilon} \rho_{0}-\rho_{0}\right\|_{B}$. This result is, unfortunately, not so useful for this map: because $\rho_{0}$ is discontinuous, $G_{\epsilon} \rho_{0}$ will not converge to $\rho_{0}$ in $\|\cdot\|_{B}$ as $\epsilon \rightarrow 0$.

\section{Numerical method.}

For this example, it is straightforward to implement the finite difference scheme of Section 2.1, with the following modifications: 


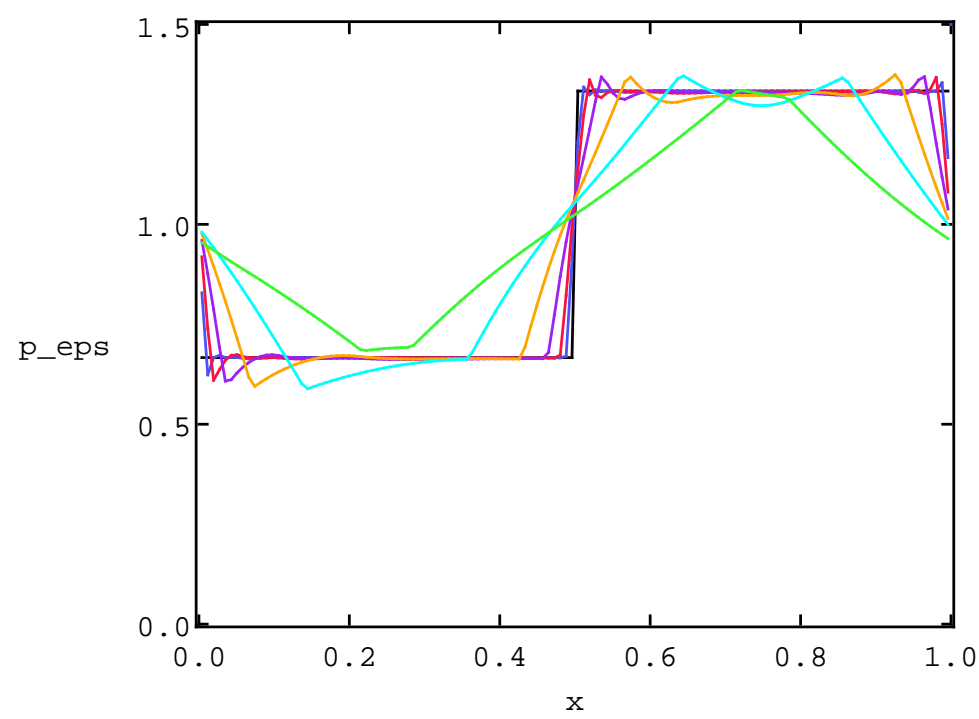

Figure 5: Invariant densities $\rho_{\epsilon}$ for (19) and its random perturbations, with $\epsilon=0$ (black curve), $0.0088,0.0176,0.0353$, $0.0707,0.141$, and 0.282 . As $\epsilon$ increases, the width of the transition region increases too.

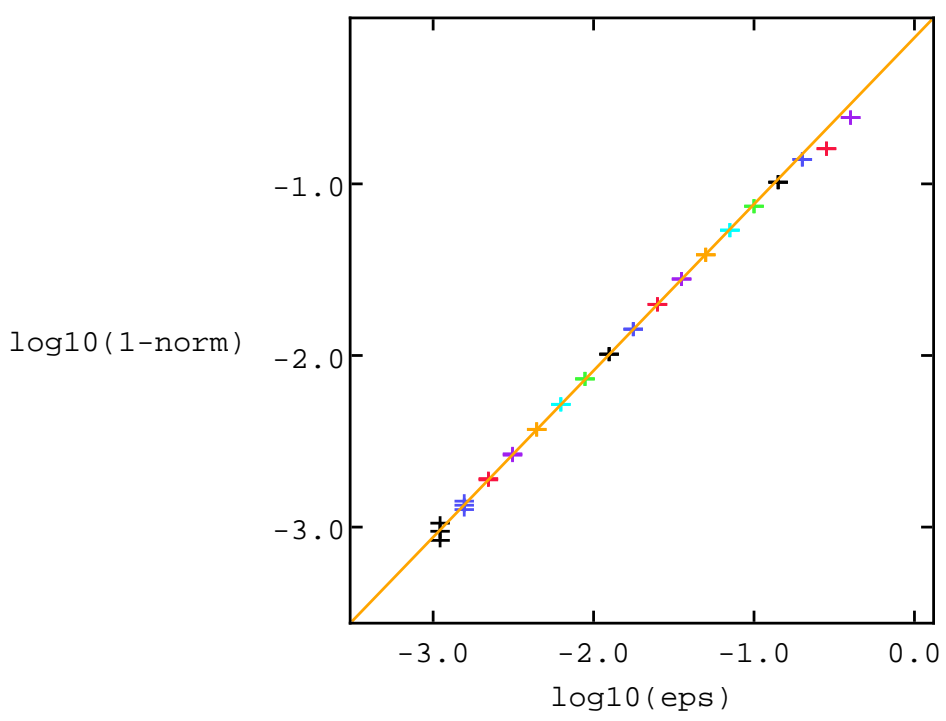

Figure 6: The differences $\left\|\rho_{\epsilon}-\rho_{0}\right\|_{1}$ and $\left\|G_{\epsilon} \rho_{0}-\rho_{0}\right\|_{1}$ as a function of $\epsilon$ on a log-log graph. The slope is $0.97 \pm$ 0.033 . The slope is calculated using least-squares regression on the interval $\epsilon \in\left[10^{-3}, 10^{-1}\right]$. Note that some of the data points have error bars which are too small to be seen on this scale. 


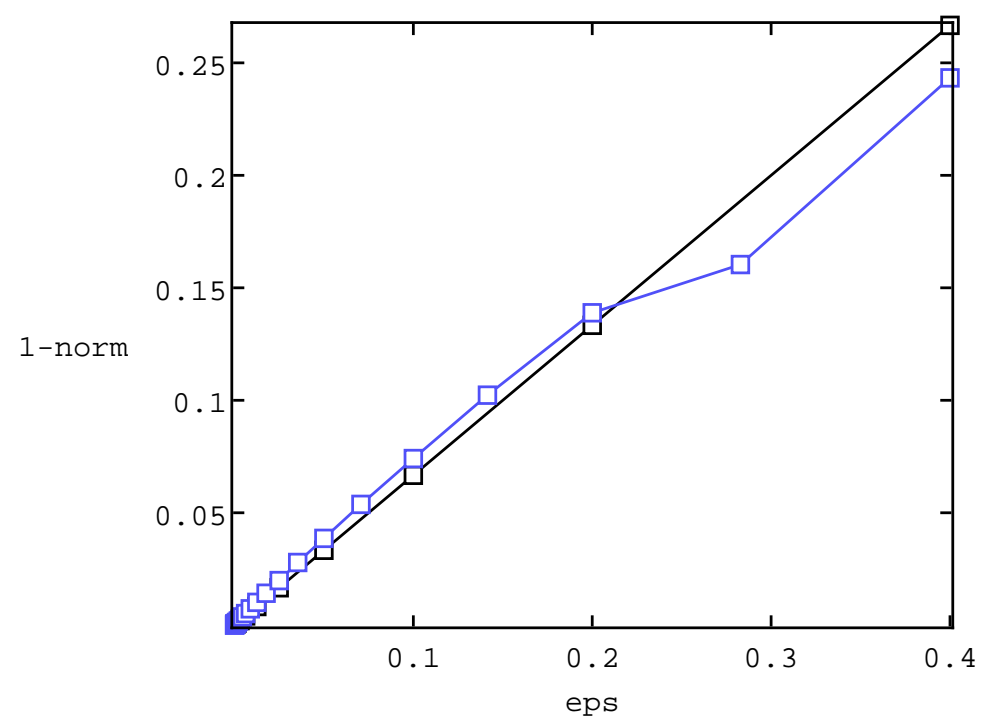

Figure 7: The differences $\left\|\rho_{\epsilon}-\rho_{0}\right\|_{1}$ and $\left\|G_{\epsilon} \rho_{0}-\rho_{0}\right\|_{1}$ as a function of $\epsilon$ on a linear graph.

1. One should avoid interpolating across discontinuities in $f$ and in $f^{\prime}$. Doing so is empirically seen to produce unacceptably large errors in the computation of $\hat{T} \hat{\rho}$. For (19), this means the polynomial interpolation of $\hat{\rho}$ should not use stencils which contain 0 or $\frac{1}{2}$ as an interior point.

2. In order to avoid interpolating across 0 or $\frac{1}{2}$, it is necessary to do one-sided interpolations, i.e. interpolate near the edge of the stencil. It is well known that one-sided polynomial interpolation on uniform grids can produce large errors. Rather than using Legendre interpolation, however, it suffices to simply increase the number of grid points and check that the resulting answer has converged numerically.

In the computations shown in this section, the polynomial interpolation of $\hat{\rho}$ utilizes a stencil with 4 points and the grid again consists of $N=10^{4}$ points. Numerical tests show that the order of accuracy lies between 3 and 4 . Thus the finite difference scheme is still sufficiently accurate to provide an efficient way to compute $\rho_{\epsilon}$.

The error bars in Figure 6 are computed by repeating the calculations with $N=5 \times 10^{3}$ points and checking the numerical convergence of the resulting estimates for $\left\|\rho_{\epsilon}-\rho_{0}\right\|_{1}$. As in $\S 2.1$, the main source of error in estimating $\left\|\rho_{\epsilon}-\rho_{0}\right\|_{1}$ comes from the trapezoid rule. The grid explicitly contains the points of discontinuity, so the trapezoid rule is still second-order accurate.

\subsection{Quadratic maps}

The third example is the quadratic map $f:[-1,1] \circlearrowleft$ :

$$
f(x)=0.9-a x^{2}, a>0 .
$$

Note that $f$ maps $[-1,1]$ into $[0.9-a, 0.9]$, so for $a \leq 1.8$ and $\epsilon \leq 0.1$, the Markov chain defined by (2) will always stay inside the interval $[-1,1]$. 


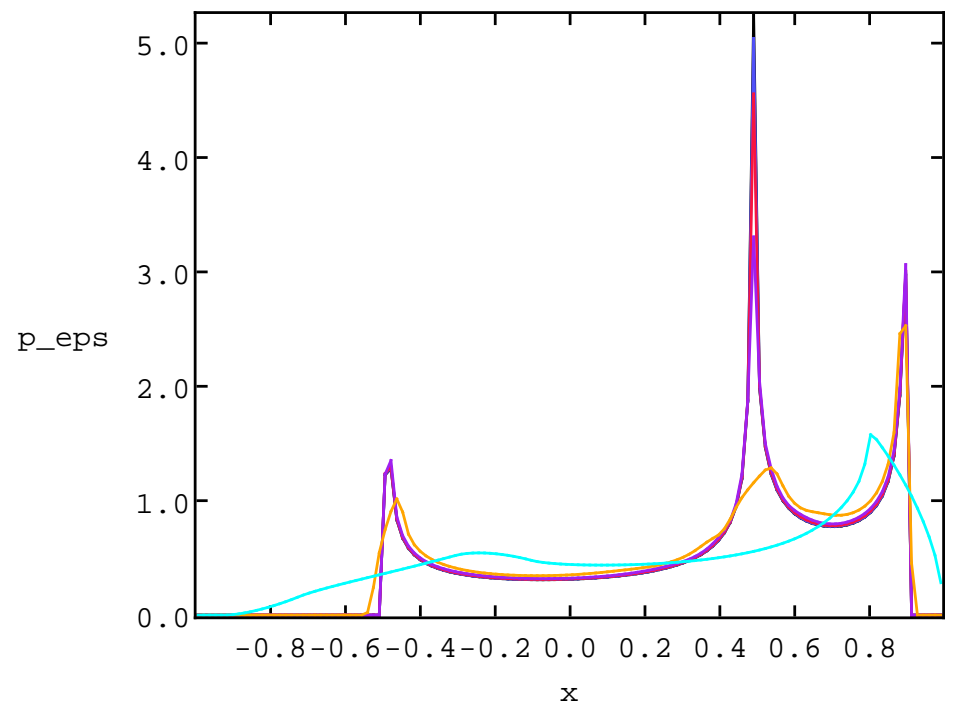

Figure 8: The invariant densities $\rho_{\epsilon}$ with increasing values of $\epsilon$. The map parameter is $a=1.7152100141023 \ldots$; the critical point is sent to an unstable fixed point at $0.489320111422868 \ldots$ in 3 steps. The noiseless density therefore has three $x^{-1 / 2}$ singularities.

Unlike the previous examples, this map has a critical point: $f^{\prime}(0)=0$. The map is contractive in a neighborhood of this critical point. Whether $f$ has a invariant density (as opposed to singular invariant measures) depends on the fate of the critical point. For example, if there exists an integer $n>0$ and a stable fixed point $x_{0}$ such that $f^{n}(0)=x_{0}$, then a positive amount of probability will collapse onto $x_{0}$ to create a $\delta$ mass there. Another scenario which can prevent the existence of an invariant density is if $f^{n}(0)$ comes close to 0 infinitely often as $n \rightarrow \infty$.

To ensure the existence of a density, it is enough to choose the parameter $a$ so that the map satisfies the Misiurewicz condition: the critical point falls into an unstable periodic orbit after a finite number of iterates. Misiurewicz proved that when this condition is satisfied, the map $f$ possesses an invariant density $\rho_{0}$ [16]. The rest of this section considers only Misiurewicz maps in the family (20). Note that even when the Misiurewicz condition is satisified, the action of $f$ creates a $x^{-1 / 2}$ singularity at each forward image $f^{n}(0)$ of the critical point 0 . Thus the invariant density $\rho_{0}$ of $f$ must contain $x^{-1 / 2}$ singularities, as one can see in Figure 8.

The Perron-Frobenius operator $T_{f}$ is known to have a spectral gap even though $f$ is not uniformly expanding [20]. This map therefore provides a more stringent test of the heuristic estimate than the previous two examples. Because the invariant density $\rho_{0}$ contains $x^{-1 / 2}$ singularities, the main effect of $G_{\epsilon}$ on $\rho_{0}$ is to mollify these singularities. This is again consistent with the picture in Figure 8. The heuristic predicts then that

$$
\left\|\rho_{\epsilon}-\rho_{0}\right\|_{1} \sim\left\|G_{\epsilon} \rho_{0}-\rho_{0}\right\|_{1} \sim \epsilon^{1 / 2} .
$$

The $L^{1}$ norms $\left\|\rho_{\epsilon}-\rho_{0}\right\|_{1}$ were calculated for $a=1.7152100141023 \ldots$ and plotted as a function of $\epsilon$ on a $\log -\log$ graph in Figure 9; the error bars mark 1 standard deviation from the computed value. Using least-squares regression on the interval $\epsilon \in\left[10^{-5}, 10^{-2.5}\right]$ to calculate the slope $\gamma$ yields $0.52 \pm 0.056$; the corresponding line is shown as a solid line. The data is consistent with the prediction that $\gamma=1 / 2$. More careful calculations are necessary to decide 


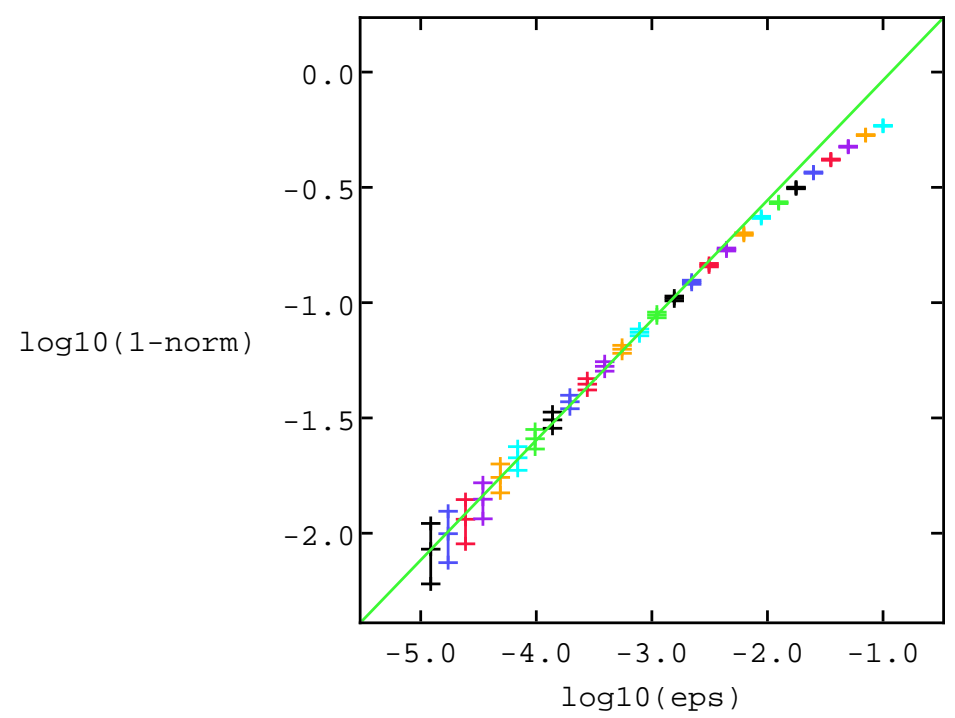

Figure 9: The differences $\left\|\rho_{\epsilon}-\rho_{0}\right\|_{1}$ and $\left\|G_{\epsilon} \rho_{0}-\rho_{0}\right\|_{1}$ as a function of $\epsilon$ on a log-log graph. The slope of the best linear fit is $0.52 \pm 0.056$; the corresponding line is shown as a solid line. The slope is calculated using least-squares regression on the interval $\epsilon \in\left[10^{-5}, 10^{-2.5}\right]$. Note that this means some of the rightmost data points are discarded. The error bars mark the mean square error in the computed value.

whether $\gamma$ is exactly $1 / 2$, as predicted by the heuristic.

The computation is repeated for another Misiurewicz parameter, $a=1.777776174649396$. Again, using leastsquares regression on the interval $\epsilon \in\left[10^{-5}, 10^{-3}\right]$ to calculate the slope $\gamma$ yields $0.46 \pm 0.050$. The results are shown in Figure 10: the data is again consistent with the claim that $\gamma=1 / 2$.

\section{Numerical method.}

Unlike the previous examples, attempts to compute invariant densities by discretizing $T_{f}$ (or $T_{\epsilon}$ ) for (20) does not work consistently: ARPACK routines will converge only for some values of $\epsilon$ and not at all for others. Sometimes ARPACK produces eigenvectors which have no obvious connection to the dynamics or to the known form of $\rho_{0}$. This sensitive dependence on the value of $\epsilon$ may be related to the extreme sensitivity of the map to the value of the parameter $a$ : Misiurewicz parameters do not form an open set; nearby values may not even have invariant probability densities. It is also possible that the structure of the spectral decomposition of $T_{f}$ is sufficiently complex that ARPACK routines could not produce well-resolved answers. ${ }^{1}$ Fortunately, because of the $x^{-1 / 2}$ singularities, the values of $\left\|\rho_{\epsilon}-\rho_{0}\right\|_{1}$ needed here are orders of magnitude larger than for the first two examples. (Compare Figures 3, 6, 9, and 10.) Furthermore, $T_{f}$ has a spectral gap, so correlations decay exponentially fast. This suggests that the usual time-averaging procedure, based on the ergodicity of $f$, can provide sufficiently accurate estimates of the probability densities $\rho_{\epsilon}$ :

1. Partition the interval $[-1,1]$ into $N$ equal-sized intervals $I_{i}$.

\footnotetext{
${ }^{1}$ In contrast, Ulam's Markov chain method should work quite well for this map. This option was not explored because it was not necessary.
} 


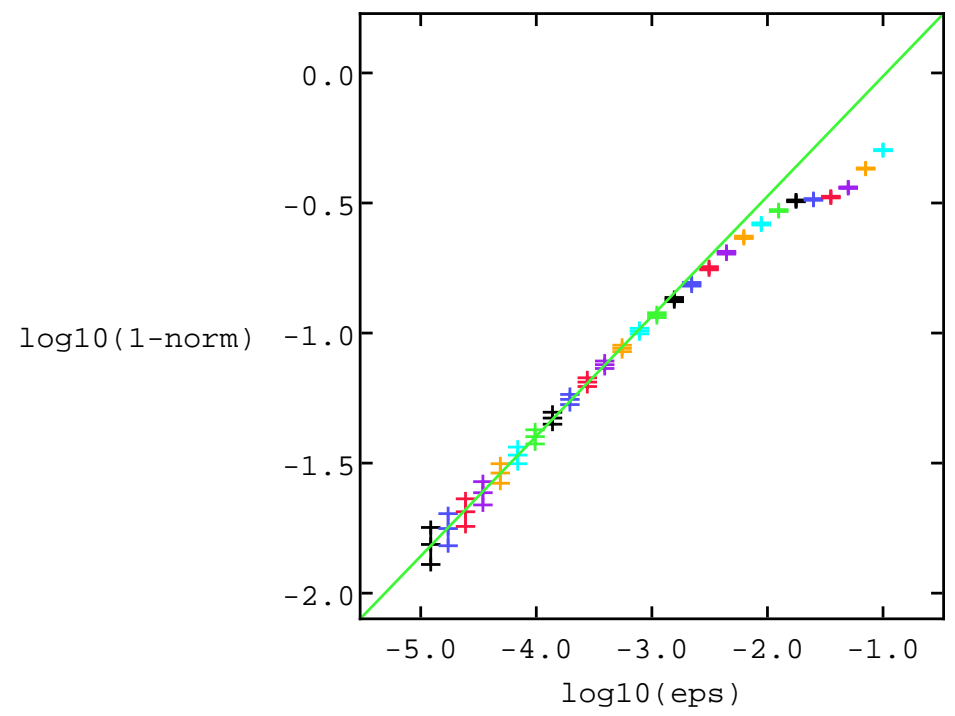

Figure 10: The differences $\left\|\rho_{\epsilon}-\rho_{0}\right\|_{1}$ and $\left\|G_{\epsilon} \rho_{0}-\rho_{0}\right\|_{1}$ as a function of $\epsilon$ on a log-log graph. The slope is $0.46 \pm$ 0.050 . The slope is calculated using least-squares regression on the interval $\epsilon \in\left[10^{-5}, 10^{-3}\right]$. The error bars mark the mean square error in the computed value.

2. Pick a random (uniform) initial condition $x_{0}$ and compute $x_{1}, x_{2}, \ldots, x_{M}$ using (2). Record the frequencies with which $x_{k}$ visits each of the intervals in the partition.

3. Let $\hat{p}_{i}$ be the relative frequency of the $i$ th interval in the partition. By the ergodic theorem, $\hat{p}_{i}$ should be approximately the probability $\int_{I_{i}} \rho_{\epsilon}(x) d x$. The $L^{1}$ distance between $\rho_{\epsilon}$ and $\rho_{0}$ can be computed by

$$
\left\|\rho_{\epsilon}-\rho_{0}\right\|_{1} \approx \sum_{i=1}^{N}\left|\hat{p}_{i}(\epsilon)-\hat{p}_{i}(0)\right| .
$$

Let us denote the estimator $\sum_{i=1}^{N}\left|\hat{p}_{i}(\epsilon)-\hat{p}_{i}(0)\right|$ by $\Phi(\epsilon)$; it provides estimates of $\left\|\rho_{\epsilon}-\rho_{0}\right\|_{1}$.

Applying the estimator $\Phi(\epsilon)$ to a set of noise amplitudes $\epsilon_{i}$ and combining the results with least squares regression yields an estimator $\hat{\gamma}$ of $\gamma$. One might expect to obtain better results by adapting the partition to better resolve the $x^{-1 / 2}$ singularities (their positions can be determined a priori: they are located on the forward images of the critical point). However, it is easier to use uniform-sized partitions, and they appear to be sufficient for this calculation.

The error analysis is standard. The estimator $\Phi(\epsilon)$ is biased because the finite partition used in Equation (22) induces a can only locate the zeros of $\rho_{\epsilon}-\rho_{0}$ up to a length scale of $N^{-1}$. More precisely, each sign change of $\rho_{\epsilon}-\rho_{0}$ contributes an error of order $N^{-2}$ to the right hand side of Equation (22). As there are only a finite number of zero crossings, the overall bias is $O\left(N^{-2}\right)$. The partition size $N$ in this calculation should be sufficiently large (see below) to make the bias negligible. The mean square error of $\hat{\gamma}$ is thus assumed to be dominated by its variance. 
The variance of $\hat{\gamma}$ can be estimated from the variance of the $\Phi(\epsilon)$ : the standard deviation of the estimated probability $\hat{p}_{i}$ is $\Delta \hat{p}_{i}=\sqrt{\frac{\hat{p}_{i}}{M}}$, where $M$ is the number of steps taken during the course of the computation. ${ }^{2}$ Summing over $i$ gives

$$
\text { standard deviation of } \Phi(\epsilon) \leq \sqrt{\frac{N}{M}} .
$$

As $\hat{\gamma}$ is linear in $\log _{10}(\Phi(\epsilon))$, its standard deviation is bounded by

$$
\frac{1}{n} \sqrt{\sum_{i=1}^{n}\left(\frac{\log _{10}\left(\epsilon_{i}\right)-\mu}{\sigma^{2}}\right)^{2} \cdot \frac{N}{M} \cdot \frac{1}{\mathbb{E}\left[\Phi\left(\epsilon_{i}\right)\right]^{2}}},
$$

where $\mu=\frac{1}{n} \sum_{i=1}^{n} \log _{10}\left(\epsilon_{i}\right), \sigma^{2}=\frac{1}{n} \sum_{i=1}^{n}\left(\log _{10}\left(\epsilon_{i}\right)-\mu\right)^{2}$, and $\frac{N}{M} \cdot \frac{1}{\mathbb{E}[\Phi(\epsilon)]^{2}}$ is an estimate of the variance of $\log _{10}(\Phi(\epsilon))$. Since the expectation values $\mathbb{E}\left[\Phi\left(\epsilon_{i}\right)\right]$ appear in the error bounds but are not available exactly, they are replaced by the estimates $\Phi\left(\epsilon_{i}\right)$ themselves in the calculations. Note that this formula assumes that the estimates $\Phi\left(\epsilon_{1}\right)$ and $\Phi\left(\epsilon_{2}\right)$ are statistically independent if $\epsilon_{1} \neq \epsilon_{2}$.

The results shown in this section have been computed using a partition of $N=2^{16}=65536$ intervals and $M=1.6 \times 10^{5} \times N \approx 10^{10}$ steps. Thus standard deviation of $\Phi(\epsilon)$ is on the order of $2.5 \times 10^{-3}$.

\section{Intermittent maps}

The examples in the previous all exhibit exponential decay of correlations. In this section I examine two examples whose Perron-Frobenius operators $T_{f}$ do not have spectral gaps and correlation functions decay algebraically. The subexponential decay is caused by parts of phase space where the map $f$ is nonexpanding. This therefore provides a model of "intermittency." See $[17,15]$.

\subsection{Circle map with neutral fixed point}

The first of the intermittent examples is the map

$$
f(x)=\left\{\begin{array}{ll}
x+2^{\alpha} x^{1+\alpha}, & 0 \leq x \leq \frac{1}{2} \\
2 x-1, & \frac{1}{2}<x<1
\end{array} .\right.
$$

This is a modification of the angle-doubling map $x \mapsto 2 x(\bmod 1)$ with a one-sided tangency to the diagonal at $x=0$ : $\lim _{x \rightarrow 0+} f^{\prime}(x)=1$. Dynamically, this means that $f(x) \approx x$ when $x$ is small and positive, so whenever $x_{k}$ lands near and to the right of the origin, many subsequent iterates are required before the trajectory "escapes" from 0 . The tangency has a significant impact on the dynamics: it can be proved that correlations decay like $n^{1-1 / \alpha}$ for this map, and that the invariant density has a $x^{-\alpha}$ singularity at $x=0$ [22]. See Figure 11.

For this example, the perturbation kernel is taken to be

$$
g(x)=\left\{\begin{array}{cc}
1, & -1 \leq x \leq 0 \\
0, & \text { otherwise }
\end{array}\right.
$$

\footnotetext{
${ }^{2}$ This assumes that the number of points in a given interval follows a Poisson distribution.
} 


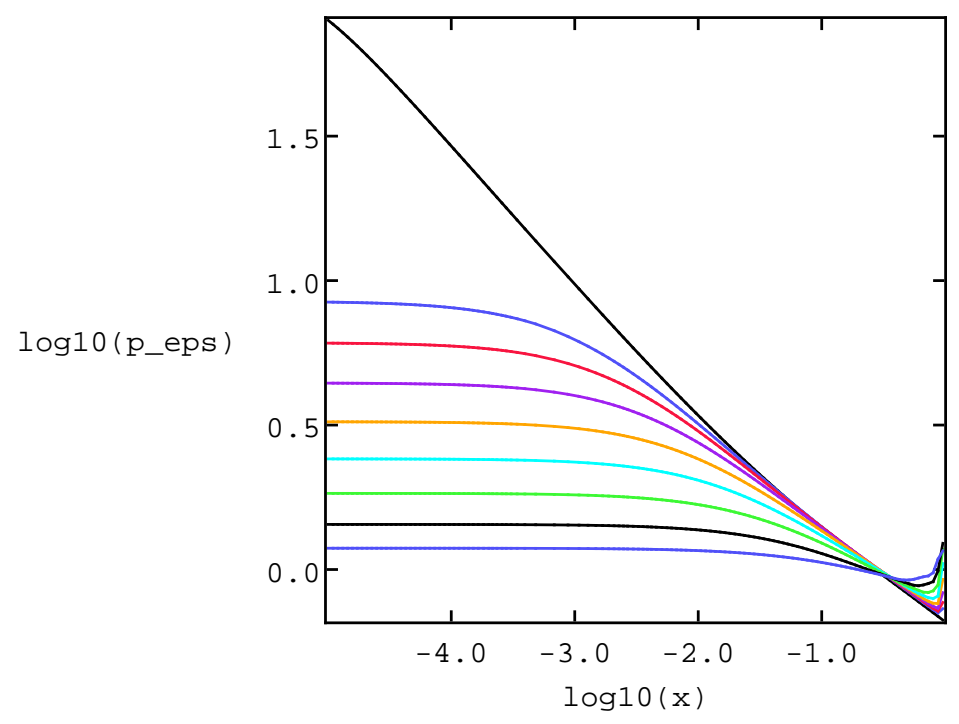

Figure 11: The invariant densities $\rho_{\epsilon}$ with increasing values of $\epsilon$. The map parameter is $\alpha=0.5$. The noiseless density $\rho_{0}$ therefore has a $x^{-0.5}$ singularity. Note that this figure uses a log-log scale.

This choice is arbitrary; using (10) does not affect the results.

As in Section 2.3, the main effect of $G_{\epsilon}$ is to smooth out the singularity in $\rho_{0}$. Thus the heuristic (8) would predict that

$$
\left\|\rho_{\epsilon}-\rho_{0}\right\|_{1} \sim\left\|G_{\epsilon} \rho_{0}-\rho_{0}\right\|_{1} \sim \epsilon^{1-\alpha}
$$

This prediction does not change when one uses the more general form of the heuristic (7) with $n>1$. However, it can be seen in Figure 12 that $\left\|\rho_{\epsilon}-\rho_{0}\right\|_{1} \nsim \epsilon^{1-\alpha}$ : in this computation $\alpha=0.5$, so we would expect $\epsilon^{0.5}$ convergence as $\epsilon \rightarrow 0$. And yet the computed exponent $\gamma$ is only $0.31 \pm 0.028$, significantly smaller than 0.5 . This result can be verified by repeating the calculation for different values of $\alpha$ : for $\alpha=0.3$, the heuristic predicts $\epsilon^{0.7}$ convergence. The real exponent is $0.53 \pm 0.056$. And for $\alpha=0.7$, the heuristic predicts $\epsilon^{0.3}$ convergence. The real exponent is $0.17 \pm 0.033$. The heuristic is qualitatively correct, though: as $\alpha$ increases, $\gamma$ decreases. Also, the computed exponents are consistent with the fact (see Section 2.1) that the heuristic always provides a lower bound for the error $\left\|\rho_{\epsilon}-\rho_{0}\right\|{ }_{1}$.

The numerical results indicate that the lack of a spectral gap can have a significant, qualitative impact on the rate of convergence of $\rho_{\epsilon}$ to $\rho_{0}$, and hence on the degree of stability of $\rho_{0}$ under random perturbations.

\section{Numerical method.}

The computations for this example again rely on the finite difference scheme of Sections 2.1 and 2.2. The only differences are:

1. As in Section 2.2, it is important to avoid interpolating across discontinuities of $f^{\prime}$. Again, this means using stencils which do not contain $x=0$ and $x=1 / 2$ as interior points. In fact, because of the singularity in $\rho_{0}$ at 0 , the origin should be excluded from the grid.

2. In order to resolve the $x^{-\alpha}$ singularity in $\rho_{0}(x)$, it is necessary to make the grid finer near 0 . The grid I use is a 


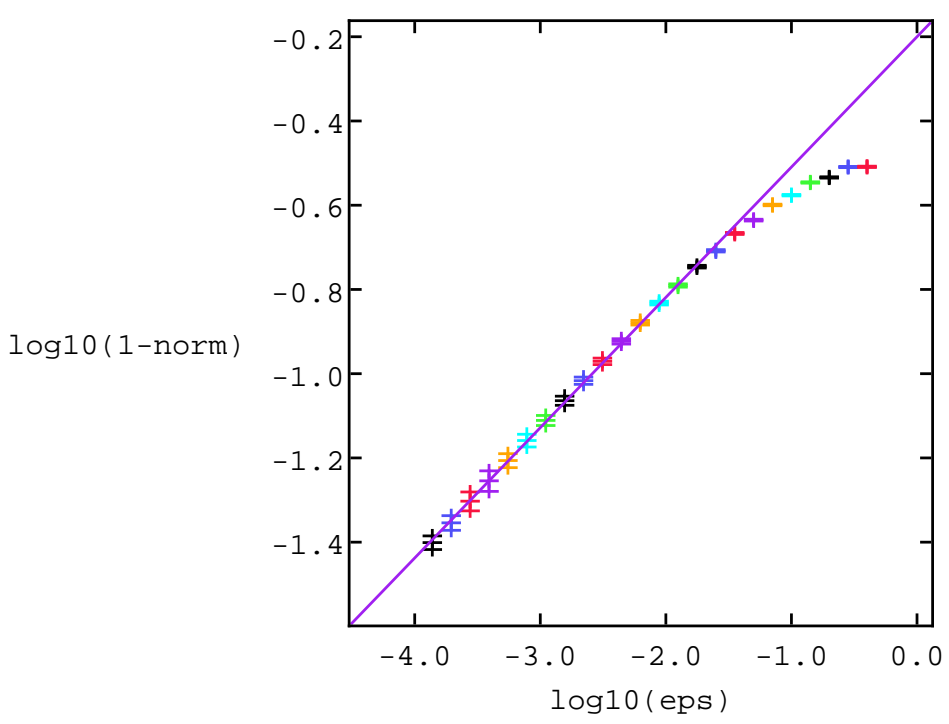

Figure 12: The differences $\left\|\rho_{\epsilon}-\rho_{0}\right\|_{1}$ and $\left\|G_{\epsilon} \rho_{0}-\rho_{0}\right\|_{1}$ as a function of $\epsilon$ on a log-log graph. The slope is $0.31 \pm$ 0.028 and is calculated using least-squares regression on the interval $\epsilon \in\left[10^{-4}, 10^{-1.5}\right]$.

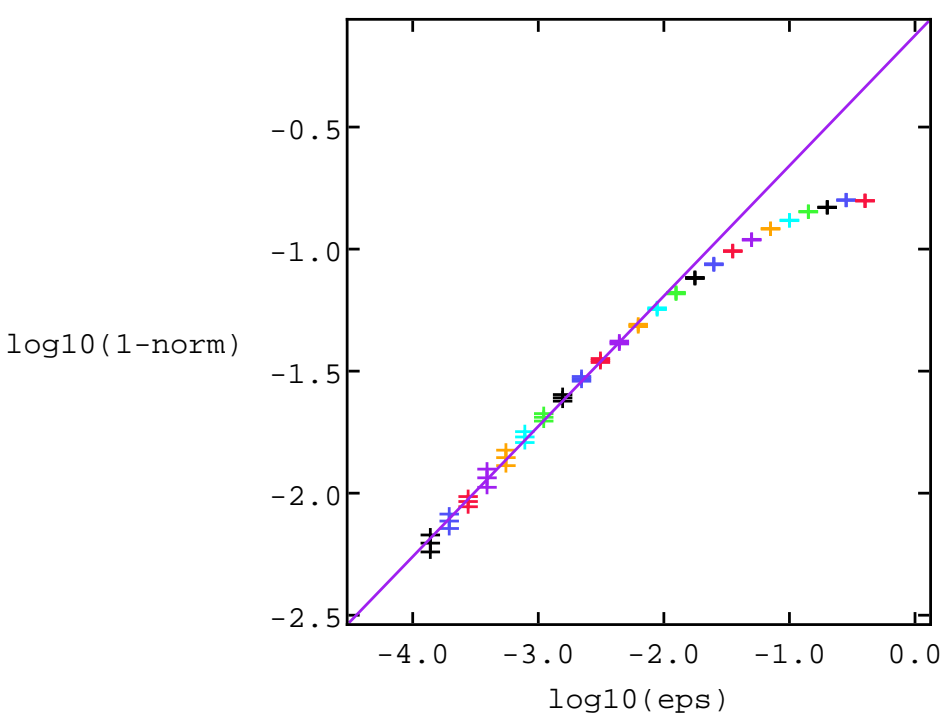

Figure 13: The differences $\left\|\rho_{\epsilon}-\rho_{0}\right\|_{1}$ and $\left\|G_{\epsilon} \rho_{0}-\rho_{0}\right\|_{1}$ as a function of $\epsilon$ on a log-log graph. The slope is $0.53 \pm$ 0.056 and is calculated using least-squares regression on the interval $\epsilon \in\left[10^{-4}, 10^{-2}\right]$. 


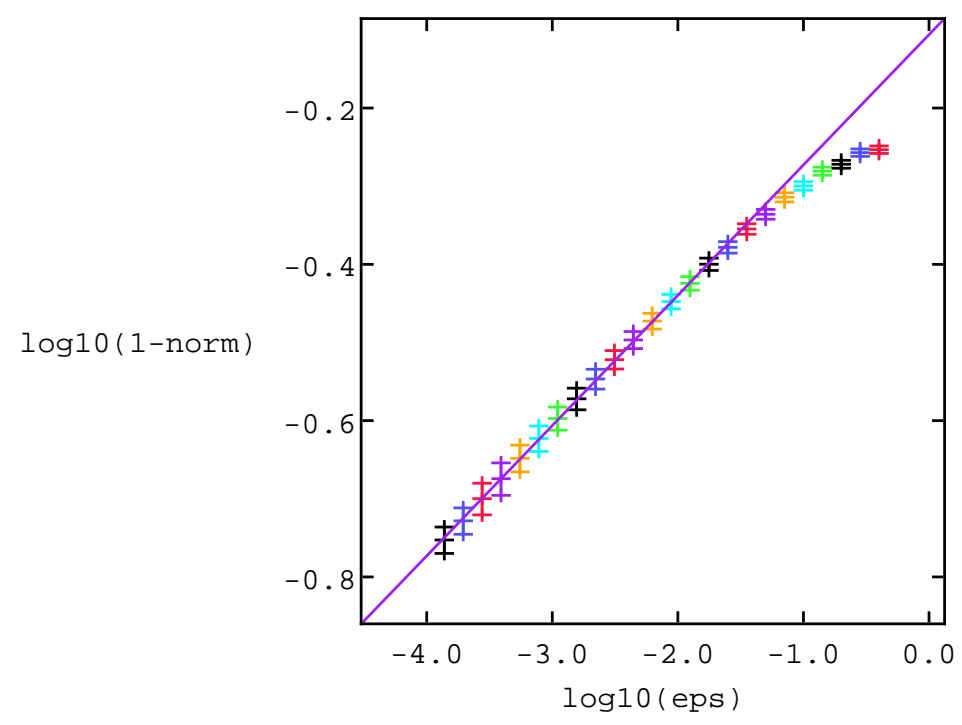

Figure 14: The differences $\left\|\rho_{\epsilon}-\rho_{0}\right\|_{1}$ and $\left\|G_{\epsilon} \rho_{0}-\rho_{0}\right\|_{1}$ as a function of $\epsilon$ on a log-log graph. The slope is $0.17 \pm$ 0.033 and is calculated using least-squares regression on the interval $\epsilon \in\left[10^{-4}, 10^{-1.5}\right]$.

hybrid between a uniform grid and one which scales like a power law: near $x=0$ the grid switches to one with points $\hat{x}_{j} \propto j^{-\alpha}, j=1,2,3, \ldots$. This scaling is the same as in Young's tower construction [22].

In the computations shown in this section, the polynomial interpolation of $\hat{\rho}$ utilizes a stencil with 6 points and the grid consists of $N=10^{4}$ points. Numerical tests show that the order of convergence is between 5 and 6 . Thus the finite difference scheme is sufficiently accurate to provide an efficient way to compute $\rho_{\epsilon}$.

\subsection{The Bunimovich stadium}

The last example is the stadium billiard: begin with a domain $\Omega \subset \mathbb{R}^{2}$ which is the union of a rectangle and two semi-circular ends, and consider the dynamics of a free point particle inside $\Omega$ which collides with $\partial \Omega$ elastically (see Figure 15). The state of the particle can therefore be represented by a point in $\Omega$ together with a unit vector. The discussion in this section focuses on the stadium map, which is the Poincare map $f$ defined by the boundary $\partial \Omega$. That is, the map $f$ takes as input a pair $(x, \theta)$, where $x \in \partial \Omega$ and $\theta$ is an angle specifying the velocity of the particle, and outputs the position and velocity of the particle after the next collision has occurred. ${ }^{3}$ Mathematically, the domain of $f$ is homeomorphic to $S^{1} \times[0, \pi]$, with the position variable being periodic. Note that unlike all other examples in this paper, the billiard map is 2-dimensional. The map $f$ also has singularities: $D f$ is discontinuous on the preimage of the vertical lines in Figure 17. The expository article by Chernov and Young [5] provides a clear survey of the statistical properties of billiards. While they do not discuss the stadium, they do explains many relevant ideas in terms of other billiard models.

\footnotetext{
${ }^{3}$ This map falls outside of the framework set up in the Introduction, but most of the general discussion there applies to this example with only minor modifications.
} 


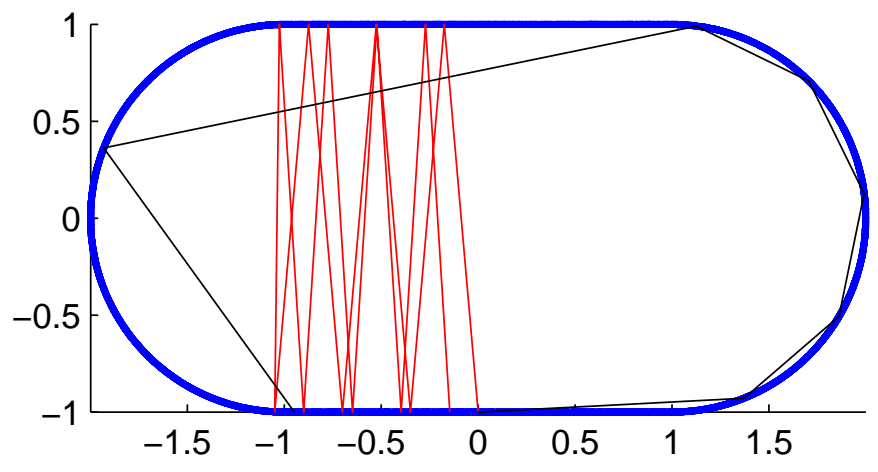

Figure 15: The stadium. Two trajectories are shown here: a perturbed "bouncing ball" orbit between the top and bottom edges, and a near "whispering gallery" orbit which wound around the right circular wing.

In order to carry out numerical calculations, a specific coordinate system is needed. The calculations described here adhere to the following coordinates: fix a reference point $p_{*}$ on $\partial \Omega$ and specify points $p$ on $\partial \Omega$ by the length $x$ of the arc subtended by $p_{*}$ and $p$ in the counterclockwise direction. The velocity vector is specified by the angle $\theta \in[0, \pi]$ between the vector and the tangent line to $\partial \Omega$, again in a counterclockwise direction. See Figure 17. In these coordinates, the billiard map $f$ preserves an invariant density $\rho_{0}(x, \theta) \propto \sin (\theta)$; the invariant density is uniform in the $x$ (arclength) variable. The pair $\left(f, \rho_{0}\right)$ is ergodic and has a positive Lyapunov exponent $[4,6]$.

For simplicity, rather than adding noise to both the $x$ and $\theta$ coordinates, only the $\theta$ coordinate is perturbed in this example. The choice of $\theta$ is natural: the geometry of the stadium suggests that small changes in $\theta$ will be magnified very quickly; perturbations in $x$ alone will not necessarily produce that effect. Furthermore, perturbations in $\theta$ destroys all metastable periodic orbits, such as the so-called "bouncing ball" orbits. This ensures that the random dynamical system (2) has a unique invariant measure. One complexity which arises in adding noise to $\theta$ alone is that the perturbation can no longer be purely additive: $\theta$ must lie between 0 and $\pi$. In order to satisfy this constraint, the following kernel is used:

$$
g_{\epsilon}\left(\theta_{\text {old }} \mapsto \theta_{\text {new }}\right)=\left\{\begin{array}{ll}
\frac{1}{\epsilon+\theta_{\text {old }}}, & 0 \leq \theta_{\text {old }}<\epsilon \\
\frac{1}{2 \epsilon}, & \epsilon \leq \theta_{\text {old }} \leq \pi-\epsilon . \\
\frac{1}{\epsilon+\pi-\theta_{\text {old }}}, & \pi-\epsilon<\theta_{\text {old }} \leq \pi
\end{array} .\right.
$$

This is a somewhat arbitrary recipe; it is not clear how much of the results in this section are due to the arbitrary nature of this specific recipe and how much is truly intrinsic to the stadium.

Figure 16 shows the difference $\rho_{\epsilon}-\rho_{0}$. The particular stadium used in the computation consists of a square $[-1,1]^{2}$ with two semi-circles of radius 1 attached. The arclength variable therefore ranges from 0 up to the perimeter $L=2 \pi+4$ of the stadium. In these coordinates, then, the vertical strips $B=[\pi / 2,2+\pi / 2] \times[0, \pi]$ and $D=$ 


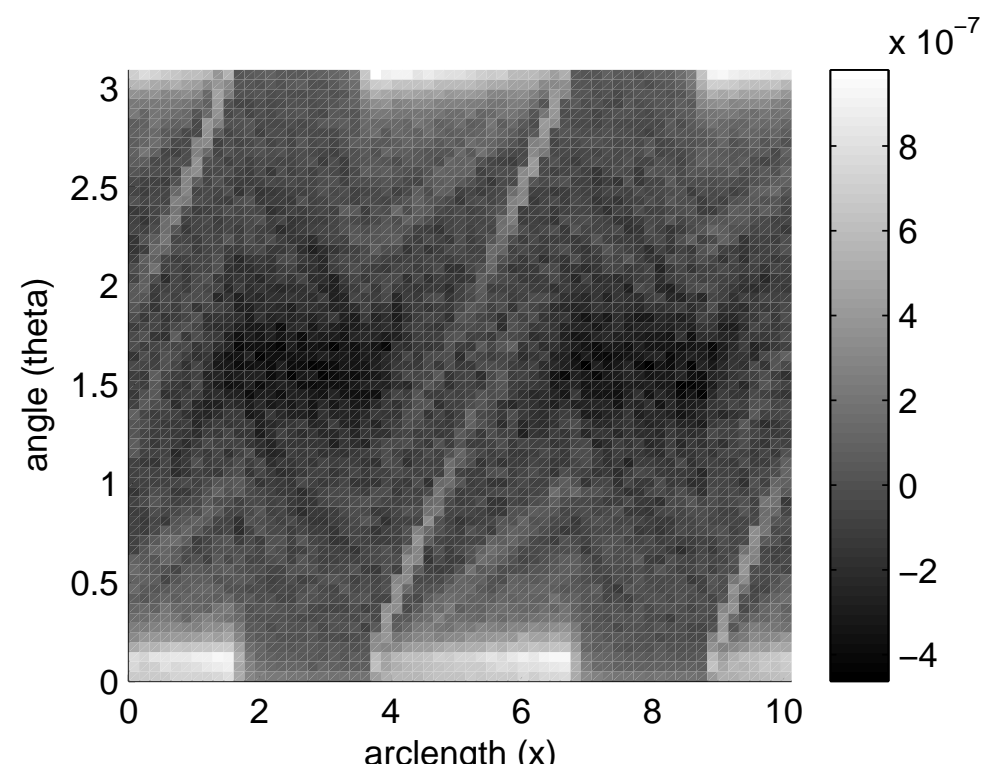

Figure 16: Level sets of $\rho_{\epsilon}-\rho_{0}$, with $\epsilon=0.1$.

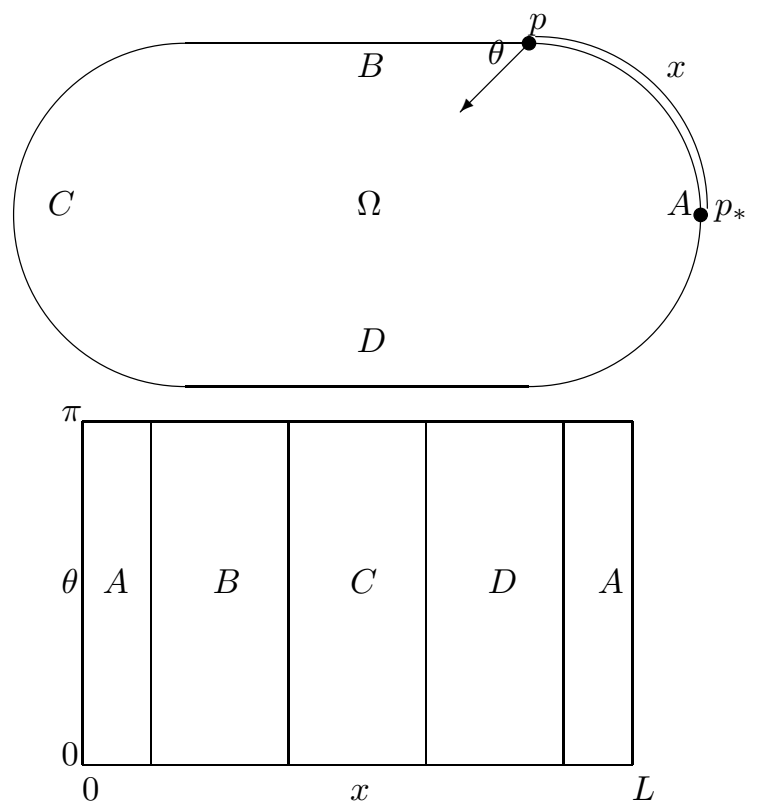

Figure 17: Illustration of the coordinate system. The angle $\theta$ ranges from 0 to $\pi$ while the arclength (position) $x$ ranges from 0 to $L$, the perimeter of the stadium $\Omega$. 


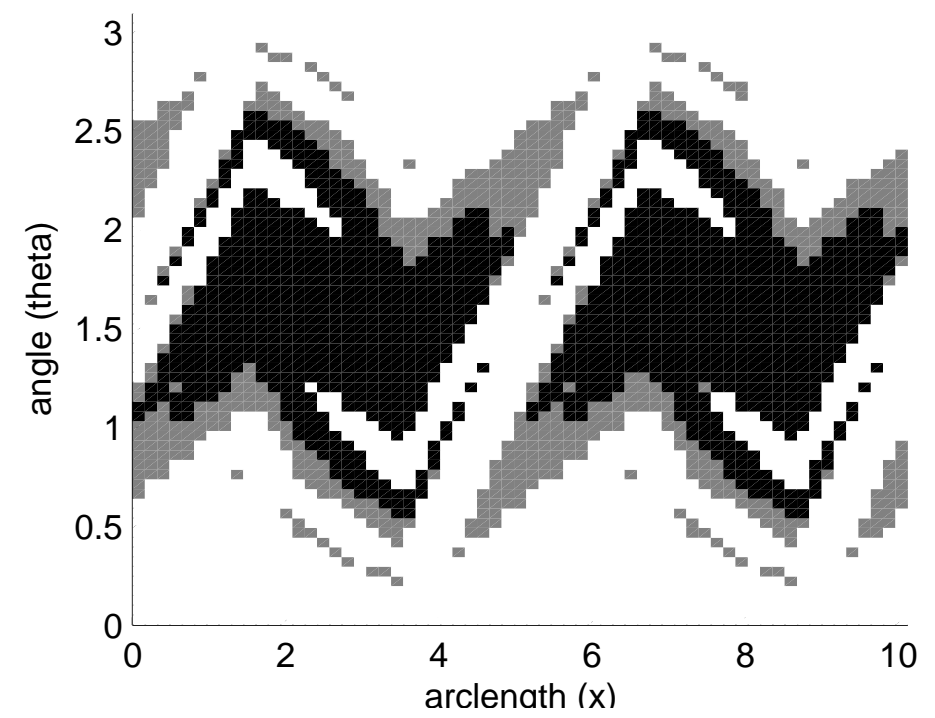

Figure 18: Dynamically-generated support of the test function $\phi_{\epsilon}$. The dark black set is $\left\{\phi_{\epsilon}=-1\right\}$, the white set $\left\{\phi_{\epsilon}=+1\right\}$, and the remainder (gray) is $\left\{\phi_{\epsilon}=0\right\}$.

$[3 \pi / 2+2,3 \pi / 2+4] \times[0, \pi]$ correspond to the flat edges of the stadium, whereas the strips $A=[-\pi / 2, \pi / 2] \times[0, \pi]$ and $C=[\pi / 2+2,3 \pi / 2+2] \times[0, \pi]$ correspond to the circular ends. As one can see, $\rho_{\epsilon}-\rho_{0}$ is more negative near the middle of the strips $B$ and $D$ and more positive near the edges of the strips $A$ and $C$. This is not surprising: the effect of the perturbation is to decrease the amount of probability near the vertical bouncing ball orbits, which correspond to the parts of $B$ and $D$ near $\theta=\frac{\pi}{2}$, and the asymmetry in the recipe (26) tends to create shallower trajectories with angles nearer 0 or $\pi$.

Because $\rho_{0}$ is smooth, the effect of applying $G_{\epsilon}$ to $\rho_{0}$ once is to smooth it out further. Thus the heuristic predicts $\left\|\rho_{\epsilon}-\rho_{0}\right\|_{1} \sim \epsilon^{2}$. In this case, it is entirely possible that the heuristic (8) does not always apply. Instead, Equation (7) may be needed with $n>1$. It is clear that, at least for $n=2$, the main effect of the singularties in $D f$ is to introduce "ridges" into $T_{\epsilon}^{2} \rho_{0}$, i.e. lines in $S^{1} \times[0, L]$ along which $T_{\epsilon}^{2} \rho_{0}$ is not differentiable in one direction. Explicit calculations show that such ridges contribute a term of $O\left(\epsilon^{2}\right)$ to $\left\|T_{\epsilon}^{2} \rho_{0}-\rho_{0}\right\|_{1}$.

\section{Numerical method and results.}

Because of the non-smoothness of the boundary $\partial \Omega$, the stadium map $f$ is also not smooth. Unlike the one-dimensional case (see Sections 2.2 and 3.1), these sets of discontinuity are no longer mere point sets but have geometric structure. This makes the direct discretization of $T_{f}$ troublesome and the finite difference method of $\S 2.1, \S 2.2$, and $\S 3.1$ difficult to apply. And, unlike the quadratic map in Section 2.3, the noiseless stadium dynamics has slow decay of correlations:

$$
\left|\int_{S^{1} \times[0, L]} \phi \cdot\left(T_{f}^{n} \psi\right) \cdot \rho_{0}-\int_{S^{1} \times[0, L]} \phi \rho_{0} \cdot \int_{S^{1} \times[0, L]} \psi \rho_{0}\right| \leq c n^{-1} .
$$

It implies that when $\epsilon$ is small but positive, the noisy dynamics will exhibit exponential decay of correlations but with a very large decay time constant. This, combined with the two-dimensional nature of the map, renders the computation 
of $\left\|\rho_{\epsilon}-\rho_{0}\right\|_{1}$ by the method of $\S 2.3$ impractical: the error of that method is proportional to the inverse square root of the number of samples per bin, which would need to be quite large in this case.

Instead, notice that the basic property (3) of the $L^{1}$ norm tells us that if we let

$$
\phi_{\epsilon}(x)=\left\{\begin{array}{cc}
+1, & \rho_{\epsilon}-\rho_{0}>0 \\
-1, & \rho_{\epsilon}-\rho_{0}<0 \\
0, & \rho_{\epsilon}-\rho_{0}=0
\end{array}\right.
$$

then

$$
\left\|\rho_{\epsilon}-\rho_{0}\right\|_{1}=\left|\int_{M} \phi_{\epsilon} \cdot \rho_{\epsilon}-\int_{M} \phi_{\epsilon} \cdot \rho_{0}\right| \cdot
$$

This suggests that approximate knowledge of the sets $E_{+}=\left\{\rho_{\epsilon}-\rho_{0}>0\right\}$ and $E_{-}=\left\{\rho_{\epsilon}-\rho_{0}<0\right\}$ will allow us to estimate $\left\|\rho_{\epsilon}-\rho_{0}\right\|_{1}$. But the geometric structure in Figure 16 gives us quite a bit of information about $E_{ \pm}$: Let $E_{+}^{0}$ be union of the small strip $[\pi / 2, \pi / 2+2] \times[0, \epsilon]$ and its symmetric images under the action of the discrete symmetry group of the stadium. The set of initial conditions $E_{+}^{0}$ generates orbits which bounce many times with shallow angles; these are the so-called "whispering gallery" orbits. Explicit calculations show that the brighter regions in Figure 16, roughly indicating $E_{+}=\left\{\rho_{\epsilon}-\rho_{0}>0\right\}$, corresponds to the union of the forward images of $E_{+}^{0}$ under the billiard map after a few (3 or 4) iterations. Similarly, let $E_{-}^{0}$ denote the union of $[-\pi / 2, \pi / 2+2] \times[\pi / 2-\epsilon, \pi / 2+\epsilon]$ and its symmetric cousins. This set of initial conditions generates "bouncing ball" orbits, and the union of the forward iterates of $E_{-}^{0}$ provides a rough approximation of $E_{-}=\left\{\rho_{\epsilon}-\rho_{0}<0\right\}$ (the dark region in Figure 16).

This allows us to construct an observable $\phi_{\epsilon}$ as follows: let $\phi_{\epsilon}$ take on the value -1 on the two sets near the midline of Figure 18, where $\rho_{\epsilon}-\rho_{0}$ is negative, and let it take on the value +1 on the sets near the boundaries, where $\rho_{\epsilon}-\rho_{0}$ is positive, and set $\phi_{\epsilon}=0$ elsewhere. The quantity

$$
\Phi(\epsilon)=\left|\iint \phi_{\epsilon}\left(\rho_{\epsilon}-\rho_{0}\right) d x d \theta\right|
$$

is then a lower bound of $\left\|\rho_{\epsilon}-\rho_{0}\right\|_{1}$. By construction, $\phi_{\epsilon}$ should maximize $\Phi(\epsilon)$ so that $\Phi(\epsilon)$ is as close to $\left\|\rho_{\epsilon}-\rho_{0}\right\|_{1}$ as possible.

The quantity $\Phi(\epsilon)$ is readily computable by averaging the values of the observable $\phi_{\epsilon}$ over a long simulated trajectory. The statistical error can be estimated in a standard way [19]: the standard deviation $\Delta \Phi(\epsilon)$ of the estimated $\Phi(\epsilon)$ is bounded above by

$$
\Delta \Phi(\epsilon) \leq \sqrt{\frac{2 \cdot \operatorname{var}\left(\phi_{\epsilon}\right) \cdot \tau_{\text {corr }}}{N}}
$$

where the autocorrelation time $\tau_{\text {corr }}=\sum_{n=0}^{\infty} C(n)$ and $C(n)$ is the autocovariance function of the observable $\phi_{\epsilon}$. The results are shown in Figure 19, where $\Phi(\epsilon)$ is plotted against $\epsilon$ on a $\log -\log$ scale. As usual, the error bars mark 1 standard deviation. The slope is approximately $1.40 \pm 0.022$ when fitted over the range $-2.5 \leq \log _{10}(\epsilon) \leq-1$. As $\Phi(\epsilon)$ only provides a lower bound on $\left\|\rho_{\epsilon}-\rho_{0}\right\|_{1}$, the main conclusion of this computation is that $\left\|\rho_{\epsilon}-\rho_{0}\right\|_{1}$ cannot decay faster than $\epsilon^{1.4}$.

For Figure 19, $\Phi(\epsilon)$ is computed, when $\epsilon>0.00625$, using a trajectory consisting of $5.1 \times 10^{8}$ steps. When $\epsilon \leq 0.00625$, the computation uses $8.1 \times 10^{9}$. This is necessary because in order to determine the exponent $\gamma$, one must compute $\log _{10}(\Phi(\epsilon))$ accurately. But the absolute error in $\log _{10}(\Phi(\epsilon))$ is proportional to the relative error in $\Phi(\epsilon)$, so it is necessary to use more steps when $\Phi(\epsilon)$ is small. 


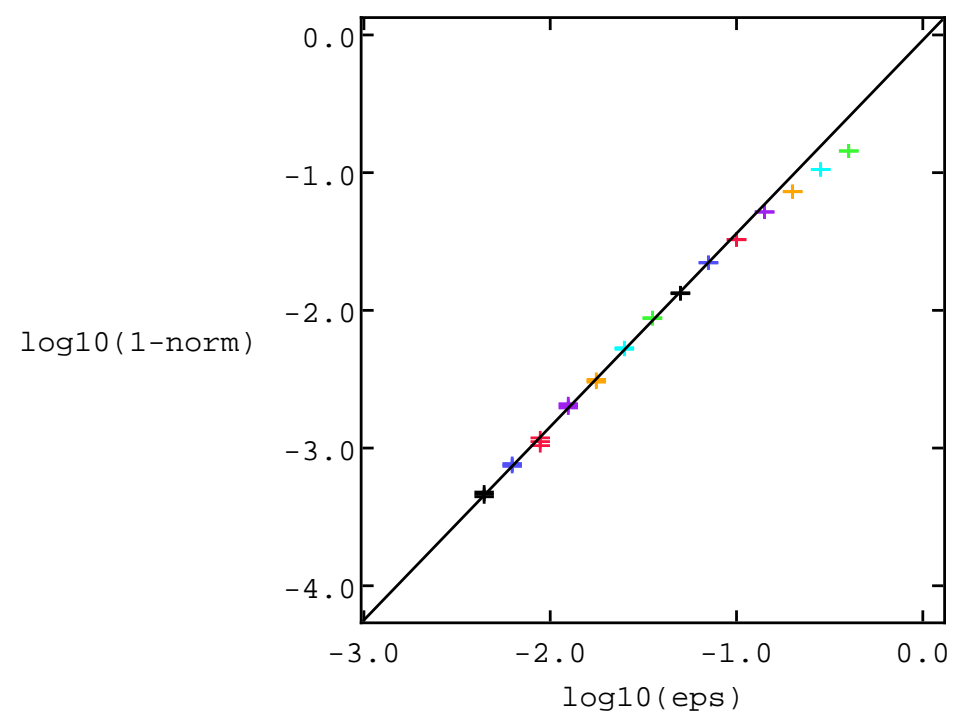

Figure 19: This figure plots $\Phi(\epsilon)=\left|\iint \phi_{\epsilon}\left(\rho_{\epsilon}-\rho_{0}\right) d x d \theta\right|$ as a function of $\epsilon$, on a log-log scale. This provides a rigorous lower bound on the norm $\left\|\rho_{\epsilon}-\rho_{0}\right\|_{1}$. The observable $\phi_{\epsilon}$ is constructed to make the two quantities as close as possible. The slope of the best linear fit is $1.40 \pm 0.022$ when least-squares regression is performed over the range $\epsilon \in\left[10^{-2.5}, 10^{-1}\right]$. This excludes points on the far right. The error bars mark 1 standard deviation. Note that some of the data points have errors which are too small to be seen on this scale.

\section{Concluding Remarks}

The calculations described in this paper lead to many intriguing questions. In addition to the question of how one might formulate and prove a precise version of the heuristic estimate (7), there is also the question of how to correctly predict the scaling of $\left\|\rho_{\epsilon}-\rho_{0}\right\|_{1}$ in simple intermittent systems. For this question, large deviations theory [8, 18] or renormalization techniques [9] may be relevant.

Invariant densities represent the most regular type of invariant measures. In most dissiptive systems, invariant measures are supported on attractors of zero measure. Among such singular invariant measures, the best-behaved are SRB measures: they represent the "nicest" invariant measures one can hope to have in dissipative chaotic dynamics. It is natural ask the corresponding question of convergence rates for SRB measures, say the rate at which $\mu_{\epsilon}$ converges to $\mu_{\mathrm{SRB}}$ in the total variation norm. The answer, however, is not so apparent. It seems sensible to conjecture that, at least in uniformly hyperbolic systems, the rate of convergence may be determined by the regularity of the conditional densities of $\mu_{\mathrm{SRB}}$ along unstable manifolds.

Another set of open questions have to do with dimension. All the examples considered in this paper exist in low-dimensional spaces. Are there other factors which can affect the rate of convergence in higher dimensions which cannot be seen in low dimensions?

The numerical calculations described in this paper employed a variety of methods. The convergence properties of these numerical methods is not yet completely understood and await deeper analysis. In particular, the extent to which a discrete transfer operator $\hat{T}$ captures the detailed spectral structure of $T_{f}$ and the effect that this has on the convergence of numerical eigenproblem routines is far from understood, as indicated by the sensitivity of the computed 
density for the Misiurewicz maps of $\S 2.2$ to changes in the parameters $a$ and $\epsilon$.

Finally, as mentioned earlier, the results described here may be relevant for numerical studies of dynamical systems with intermittent, metastable behavior. Noise can help reduce initialization bias in long-time numerical simulations while introducing controllable errors. This may be particularly useful in intermittent systems of the type examined in Sections 3.1 and 3.2. A first step in this direction was made in [14], but a systematic exploration is required to understand these ideas. In particular, to carry out this idea in practice will require algorithms which can cope with the additional complexity of separatrices and multiple ergodic components.

\section{Acknowledgements}

It is a pleasure to thank Lai-Sang Young for her generous help with this project. I am also grateful to Alex Barnett, Toufic Suidan, and George Zaslavsky for helping to improve the exposition and for many helpful and pleasant conversations. This work is supported by the National Science Foundation through a Mathematical Sciences Research Postdoctoral Fellowship.

\section{References}

[1] V. BALAdI, Positive Transfer Operators and Decay of Correlations, World Scientific, 2000.

[2] V. Baladi AND L.-S. Young, On the spectra of randomly perturbed expanding maps, Communications in Mathematical Physics, 156 (1993).

[3] M. Blank AND G. Keller, Random perturbations of chaotic dynamical systems: stability of the spectrum, Nonlinearity, 11 (1998), pp. 1351-1364.

[4] L. A. Bunimovič, The ergodic properties of certain billiards, Funkcional. Anal. i Priložen., 8 (1974), pp. $73-$ 74.

[5] N. Chernov And L. S. Young, Decay of correlations for Lorentz gases and hard balls, in Hard ball systems and the Lorentz gas, vol. 101 of Encyclopaedia Math. Sci., Springer, Berlin, 2000, pp. 89-120.

[6] V. J. Donnay, Using integrability to produce chaos: billiards with positive entropy, Communications in Mathematical Physics, 141 (1991).

[7] J.-P. ECKMANn AND D. RuelLe, Ergodic theory of chaos and strange attractors, Reviews of Modern Physics, 57 (1985), pp. 617-656.

[8] M. I. Freidlin And A. D. Wentzell, Random Perturbations of Dynamical Systems, Springer-Verlag, 1998.

[9] J. E. Hirsch, M. NaUenberg, AND D. J. Scalapino, Intermittency in the presence of noise: a renormalization group formulation, Physics Letters, 87A (1982).

[10] M. Keane, R. Murray, And L.-S. Young, Computing invariant measures for expanding circle maps, Nonlinearity, 11 (1998), pp. 27-46.

[11] Y. I. KIFER, Small random perturbations of certain smooth dynamical systems, Izv. Akad. Nauk SSSR Ser. Mat., 38 (1974), pp. 1091-1115. 
[12] A. LASOTA AND J. A. YORKE, On the existence of invariant measures for piecewise monotonic transformations, Transactions of the American Mathematical Society, 186 (1973), pp. 481-488.

[13] R. B. LehouCQ, D. C. Sorensen, And C. YAng, ARPACK User's Guide: Solution of Large-Scale Eigenvalue Problems with Implicitly Restarted Arnoldi Methods, SIAM, 1998.

[14] K. K. LIN, Random perturbations of SRB measures and numerical studies of chaotic dynamics, PhD thesis, University of California at Berkeley, 2003. Lawrence Berkeley National Lab Technical Report 53522.

[15] C. Liverani, B. Saussol, And S. Vaienti, A probabilistic approach to intermittency, Ergodic Theory \& Dynamical Systems, 19 (1999), pp. 671-685.

[16] M. MisiurewCIZ, Absolutely continuous measures for certain maps of an interval, Publications Mathématiques de l'IHÉS, 53 (1981), pp. 17-51.

[17] Y. Pomeau And P. Manneville, Intermittent transition to turbulence in dissipative dynamical systems, Communications in Mathematical Physics, 74 (1980), pp. 189-197.

[18] P. Reimann And P. TAlkner, Invariant densities for noisy maps, Physical Review A, 44 (1991), pp. 63486363.

[19] A. D. SoKAL, Monte Carlo methods in statistical mechanics: Foundations and new algorithms, in Functional Integration (Cargèse, 1996), vol. 361 of NATO Adv. Sci. Inst. Ser. B Phys., Plenum, 1997, pp. 131-192.

[20] L.-S. Young, Decay of correlations for certain quadratic maps, Communications in Mathematical Physics, 146 (1992), pp. 123-138.

[21] - Ergodic theory of differentiable dynamical systems, in Real and Complex Dynamics, vol. 464 of NATO Adv. Sci. Inst. Ser. C Math. Phys. Sci. (Hillerød, 1993), Kluwer Academic Publishers, 1995, pp. $293-336$.

[22] - Recurrence times and rates of mixing, Israel Journal of Mathematics, (1999). 